\title{
A FINITE-DIMENSIONAL LIE ALGEBRA ARISING FROM A NICHOLS ALGEBRA OF DIAGONAL TYPE (RANK 2)
}

\author{
NICOLÁS ANDRUSKIEWITSCH, IVÁN ANGIONO, FIORELA ROSSI BERTONE
}

\begin{abstract}
Let $\mathcal{B}_{\mathfrak{q}}$ be a finite-dimensional Nichols algebra of diagonal type corresponding to a matrix $\mathfrak{q} \in \mathbf{k}^{\theta \times \theta}$. Let $\mathcal{L}_{\mathfrak{q}}$ be the Lusztig algebra associated to $\mathcal{B}_{\mathfrak{q}}$ AAR. We present $\mathcal{L}_{\mathfrak{q}}$ as an extension (as braided Hopf algebras) of $\mathcal{B}_{\mathfrak{q}}$ by $\mathfrak{Z}_{\mathfrak{q}}$ where $\mathfrak{Z}_{\mathfrak{q}}$ is isomorphic to the universal enveloping algebra of a Lie algebra $\mathfrak{n}_{\mathfrak{q}}$. We compute the Lie algebra $\mathfrak{n}_{\mathfrak{q}}$ when $\theta=2$.
\end{abstract}

\section{INTRODUCTION}

1.1. Let $\mathbf{k}$ be a field, algebraically closed and of characteristic zero. Let $\theta \in \mathbb{N}, \mathbb{I}=\mathbb{I}_{\theta}:=\{1,2, \ldots, \theta\}$. Let $\mathfrak{q}=\left(q_{i j}\right)_{i, j \in \mathbb{I}}$ be a matrix with entries in $\mathbf{k}^{\times}, V$ a vector space with a basis $\left(x_{i}\right)_{i \in \mathbb{I}}$ and $c^{\mathfrak{q}} \in G L(V \otimes V)$ be given by

$$
c^{\mathfrak{q}}\left(x_{i} \otimes x_{j}\right)=q_{i j} x_{j} \otimes x_{i}, \quad i, j \in \mathbb{I} .
$$

Then $\left(c^{\mathfrak{q}} \otimes \mathrm{id}\right)\left(\mathrm{id} \otimes c^{\mathfrak{q}}\right)\left(c^{\mathfrak{q}} \otimes \mathrm{id}\right)=\left(\mathrm{id} \otimes c^{\mathfrak{q}}\right)\left(c^{\mathfrak{q}} \otimes \mathrm{id}\right)\left(\mathrm{id} \otimes c^{\mathfrak{q}}\right)$, i.e. $\left(V, c^{\mathfrak{q}}\right)$ is a braided vector space and the corresponding Nichols algebra $\mathcal{B}_{\mathfrak{q}}:=\mathcal{B}(V)$ is called of diagonal type. Recall that $\mathcal{B}_{\mathfrak{q}}$ is the image of the unique map of braided Hopf algebras $\Omega: T(V) \rightarrow T^{c}(V)$ from the free associative algebra of $V$ to the free associative coalgebra of $V$, such that $\Omega_{\mid V}=\mathrm{id}_{V}$. For unexplained terminology and notation, we refer to [AS].

Remarkably, the explicit classification of all $\mathfrak{q}$ such that $\operatorname{dim} \mathcal{B}_{\mathfrak{q}}<\infty$ is known [H2] (we recall the list when $\theta=2$ in Table 1). Also, for every $\mathfrak{q}$ in the list of [H2], the defining relations are described in [A2, A3].

1.2. Assume that $\operatorname{dim} \mathcal{B}_{\mathfrak{q}}<\infty$. Two infinite dimensional graded braided Hopf algebras $\widetilde{\mathcal{B}}_{\mathfrak{q}}$ and $\mathcal{L}_{\mathfrak{q}}$ (the Lusztig algebra of $V$ ) were introduced and studied in $\left[\mathrm{A3}, \mathrm{A5}\right.$, respectively $\mathrm{AAR}$. Indeed, $\widetilde{\mathcal{B}}_{\mathfrak{q}}$ is a pre-Nichols, and $\mathcal{L}_{\mathfrak{q}}$ a post-Nichols, algebra of $V$, meaning that $\widetilde{\mathcal{B}}_{\mathfrak{q}}$ is intermediate between $T(V)$ and $\mathcal{B}_{\mathfrak{q}}$, while $\mathcal{L}_{\mathfrak{q}}$ is intermediate between $\mathcal{B}_{\mathfrak{q}}$ and $T^{c}(V)$. This is summarized

2000 Mathematics Subject Classification. 16W30.

The work was partially supported by CONICET, FONCyT-ANPCyT, Secyt (UNC). 
in the following commutative diagram:

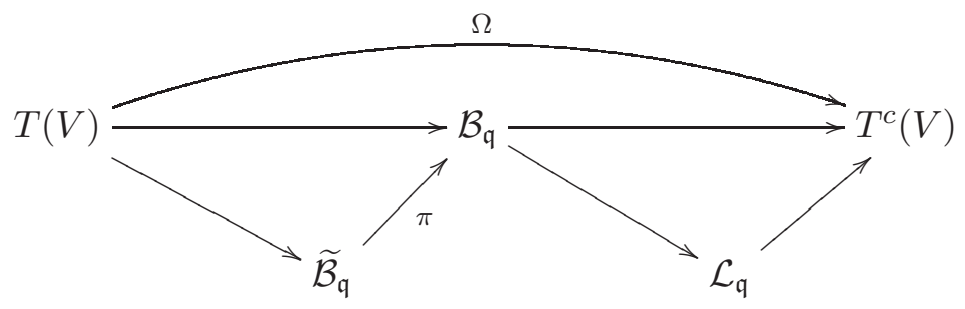

The algebras $\widetilde{\mathcal{B}}_{\mathfrak{q}}$ and $\mathcal{L}_{\mathfrak{q}}$ are generalizations of the positive parts of the De Concini-Kac-Procesi quantum group, respectively the Lusztig quantum divided powers algebra. The distinguished pre-Nichols algebra $\widetilde{\mathcal{B}}_{\mathfrak{q}}$ is defined discarding some of the relations in [A3], while $\mathcal{L}_{\mathfrak{q}}$ is the graded dual of $\widetilde{\mathcal{B}}_{\mathfrak{q}}$.

1.3. The following notions are discussed in Section 2, Let $\Delta_{+}^{\mathfrak{q}}$ be the generalized positive root system of $\mathcal{B}_{\mathfrak{q}}$ and let $\mathfrak{O}_{\mathfrak{q}} \subset \Delta_{+}^{\mathfrak{q}}$ be the set of Cartan roots of $\mathfrak{q}$. Let $x_{\beta}$ be the root vector associated to $\beta \in \Delta_{+}^{\mathfrak{q}}$, let $N_{\beta}=\operatorname{ord} q_{\beta \beta}$ and let $Z_{\mathfrak{q}}$ be the subalgebra of $\widetilde{\mathcal{B}}_{\mathfrak{q}}$ generated by $x_{\beta}^{N_{\beta}}, \beta \in \mathfrak{O}_{\mathfrak{q}}$. By A5, Theorems $4.10,4.13], Z_{\mathfrak{q}}$ is a braided normal Hopf subalgebra of $\widetilde{\mathcal{B}}_{\mathfrak{q}}$ and $Z_{\mathfrak{q}}=\operatorname{co} \pi \widetilde{\mathcal{B}}_{\mathfrak{q}}$. Actually, $Z_{\mathfrak{q}}$ is a true commutative Hopf algebra provided that

$$
q_{\alpha \beta}^{N_{\beta}}=1, \quad \forall \alpha, \beta \in \mathfrak{O}_{\mathfrak{q}} .
$$

Let $\mathfrak{Z}_{\mathfrak{q}}$ be the graded dual of $Z_{\mathfrak{q}}$; under the assumption (1) $\mathfrak{Z}_{\mathfrak{q}}$ is a cocommutative Hopf algebra, hence it is isomorphic to the enveloping algebra $\mathcal{U}\left(\mathfrak{n}_{\mathfrak{q}}\right)$ of the Lie algebra $\mathfrak{n}_{\mathfrak{q}}:=\mathcal{P}\left(\mathfrak{Z}_{\mathfrak{q}}\right)$. We show in Section 3 that $\mathcal{L}_{\mathfrak{q}}$ is an extension (as braided Hopf algebras) of $\mathcal{B}_{\mathfrak{q}}$ by $\mathfrak{Z}_{\mathfrak{q}}$ :

$$
\mathcal{B}_{\mathfrak{q}} \stackrel{\pi^{*}}{\hookrightarrow} \mathcal{L}_{\mathfrak{q}} \stackrel{\iota^{*}}{\rightarrow} \mathfrak{Z}_{\mathfrak{q}} \cdot
$$

The main result of this paper is the determination of the Lie algebra $\mathfrak{n}_{\mathfrak{q}}$ when $\theta=2$ and the generalized Dynkin diagram of $\mathfrak{q}$ is connected.

Theorem 1.1. Assume that $\operatorname{dim} \mathcal{B}_{\mathfrak{q}}<\infty$ and $\theta=2$. Then $\mathfrak{n}_{\mathfrak{q}}$ is either 0 or isomorphic to $\mathfrak{g}^{+}$, where $\mathfrak{g}$ is a finite-dimensional semisimple Lie algebra listed in the last column of Table 1 .

Assume that there exists a Cartan matrix $\mathbf{a}=\left(a_{i j}\right)$ of finite type, that becomes symmetric after multiplying with a diagonal $\left(d_{i}\right)$, and a root of unit $q$ of odd order (and relatively prime to 3 if $\mathbf{a}$ is of type $G_{2}$ ) such that $q_{i j}=q^{d_{i} a_{i j}}$ for all $i, j \in \mathbb{I}$. Then (2) encodes the quantum Frobenius homomorphism defined by Lusztig and Theorem 1.1 is a result from [L].

The penultimate column of Table 1 indicates the type of $\mathfrak{q}$ as established in [AA]. Thus, we associate Lie algebras in characteristic zero to some contragredient Lie (super)algebras in positive characteristic. In a forthcoming paper we shall compute the Lie algebra $\mathfrak{n}_{\mathfrak{q}}$ for $\theta>2$. 


\begin{tabular}{|c|c|c|c|c|}
\hline Row & I Generalized Dynkin diagrams & parameters & Type of $\mathcal{B}_{\mathfrak{q}}$ & $\mathfrak{n}_{\mathfrak{q}} \simeq \mathfrak{g}^{+}$ \\
\hline 1 & $\stackrel{q}{q} \stackrel{q}{ }^{-1} \quad q$ & $q \neq 1$ & Cartan $A$ & $A_{2}$ \\
\hline 2 & \begin{tabular}{cccccc}
$q$ & $q^{-1}$ & -1 & -1 & $q$ & -1 \\
\hdashline & - & $\bigcirc$ & $\bigcirc$ & -
\end{tabular} & $q \neq \pm 1$ & Super $A$ & $A_{1}$ \\
\hline 3 & 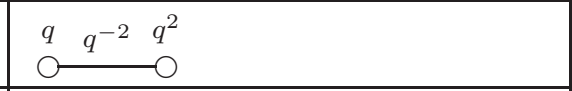 & $q \neq \pm 1$ & Cartan $B$ & $B_{2}$ \\
\hline 4 & 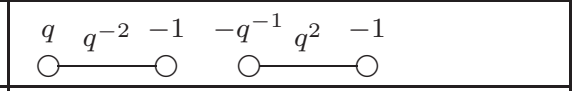 & $q \notin \mathbb{G}_{4}$ & Super $B$ & $A_{1} \oplus A_{1}$ \\
\hline 5 & 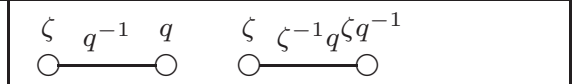 & $\zeta \in \mathbb{G}_{3} \not \supset q$ & $\mathfrak{b} \mathfrak{r}(2, a)$ & $A_{1} \oplus A_{1}$ \\
\hline 6 & \begin{tabular}{cccc}
$\zeta$ & $-\zeta$ & -1 & $\zeta^{-1}-\zeta^{-1}-1$ \\
\hdashline & $\bigcirc$
\end{tabular} & $\zeta \in \mathbb{G}_{3}^{\prime}$ & Standard $B$ & 0 \\
\hline 7 & 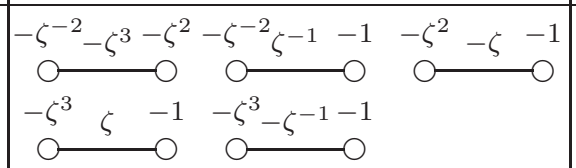 & $\zeta \in \mathbb{G}_{12}^{\prime}$ & $\mathfrak{u} \mathfrak{f} \mathfrak{o}(7)$ & 0 \\
\hline 8 & 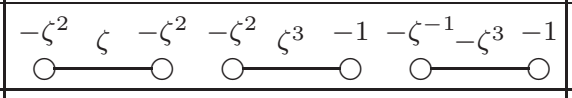 & $\zeta \in \mathbb{G}_{12}^{\prime}$ & $\mathfrak{u} \mathfrak{f} \mathfrak{o}(8)$ & $A_{1}$ \\
\hline 9 & 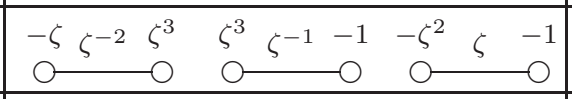 & $\zeta \in \mathbb{G}_{9}^{\prime}$ & $\mathfrak{b r j}(2 ; 3)$ & $A_{1} \oplus A_{1}$ \\
\hline 10 & \begin{tabular}{crr}
$q$ & $q^{-3}$ & $q^{3}$ \\
\hdashline & & $\bigcirc$ \\
\end{tabular} & $q \notin \mathbb{G}_{2} \cup \mathbb{G}_{3}$ & Cartan $G_{2}$ & $G_{2}$ \\
\hline 11 & 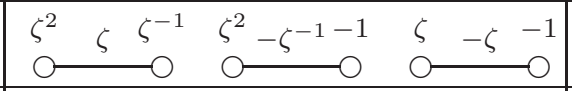 & $\zeta \in \mathbb{G}_{8}^{\prime}$ & Standard $G_{2}$ & $A_{1} \oplus A_{1}$ \\
\hline 12 & 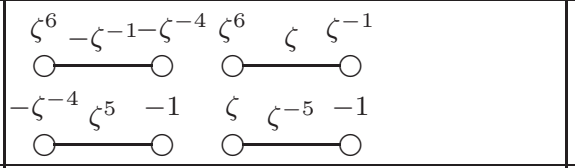 & $\zeta \in \mathbb{G}_{24}^{\prime}$ & $\mathfrak{u} \mathfrak{f o}(9)$ & $A_{1} \oplus A_{1}$ \\
\hline 13 & 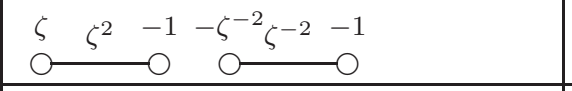 & $\zeta \in \mathbb{G}_{5}^{\prime}$ & $\mathfrak{b} \mathfrak{r j}(2 ; 5)$ & $B_{2}$ \\
\hline 14 & \begin{tabular}{cccc}
$\zeta$ & $\zeta^{-3}-1$ & $-\zeta$ & $-\zeta^{-3}-1$ \\
$\bigcirc$ & $\bigcirc$ & $\bigcirc$ \\
$-\zeta^{-2}$ & $\zeta^{3}$ & -1 & $-\zeta^{-2}-\zeta^{3}-1$ \\
\hdashline & $\bigcirc$ \\
\hdashline
\end{tabular} & $\zeta \in \mathbb{G}_{20}^{\prime}$ & $\mathfrak{u f \mathfrak { o }}(10)$ & $A_{1} \oplus A_{1}$ \\
\hline 15 & \begin{tabular}{lll}
$-\zeta-\zeta^{-3} \zeta^{5}$ & $\zeta^{3}$ & $-\zeta^{4-\zeta^{-4}}$ \\
$\bigcirc$ & $\bigcirc$ & $\bigcirc$ \\
$\zeta^{5}-\zeta^{-2}-1$ & $\zeta^{3}-\zeta^{2}-1$ \\
$\bigcirc$ & $\bigcirc$ \\
\hdashline & $\bigcirc$
\end{tabular} & $\zeta \in \mathbb{G}_{15}^{\prime}$ & $\mathfrak{u} \mathfrak{f} \mathfrak{o}(11)$ & $A_{1} \oplus A_{1}$ \\
\hline 16 & $\stackrel{-\zeta-\zeta^{-3}-1}{-\zeta^{-2}-\zeta^{3}-1}$ & $\zeta \in \mathbb{G}_{7}^{\prime}$ & $\mathfrak{u f \mathfrak { o }}(12)$ & $G_{2}$ \\
\hline
\end{tabular}

TABLE 1. Lie algebras arising from Dynkin diagrams of rank 2. 
1.4. The paper is organized as follows. We collect the needed preliminary material in Section 2. Section 3 is devoted to the exactness of (2). The computations of the various $\mathfrak{n}_{\mathfrak{q}}$ is the matter of Section 4. We denote by $\mathbb{G}_{N}$ the group of $N$-th roots of 1 , and by $\mathbb{G}_{N}^{\prime}$ its subset of primitive roots.

\section{Preliminaries}

2.1. The Nichols algebra, the distinguished-pre-Nichols algebra and the Lusztig algebra. Let $\mathfrak{q}$ be as in the Introduction and let $\left(V, c^{\mathfrak{q}}\right)$ be the corresponding braided vector space of diagonal type. We assume from now on that $\mathcal{B}_{\mathfrak{q}}$ is finite-dimensional. Let $\left(\alpha_{j}\right)_{j \in \mathbb{I}}$ be the canonical basis of $\mathbb{Z}^{\theta}$. Let $\mathbf{q}: \mathbb{Z}^{\theta} \times \mathbb{Z}^{\theta} \rightarrow \mathbf{k}^{\times}$be the $\mathbb{Z}$-bilinear form associated to the matrix $\mathfrak{q}$, i.e. $\mathbf{q}\left(\alpha_{j}, \alpha_{k}\right)=q_{j k}$ for all $j, k \in \mathbb{I}$. If $\alpha, \beta \in \mathbb{Z}^{\theta}$, we set $q_{\alpha \beta}=\mathbf{q}(\alpha, \beta)$. Consider the matrix $\left(c_{i j}^{\mathfrak{q}}\right)_{i, j \in \mathbb{I}}, c_{i j} \in \mathbb{Z}$ defined by $c_{i i}^{\mathfrak{q}}=2$,

$$
c_{i j}^{\mathfrak{q}}:=-\min \left\{n \in \mathbb{N}_{0}:(n+1)_{q_{i i}}\left(1-q_{i i}^{n} q_{i j} q_{j i}\right)=0\right\}, \quad i \neq j .
$$

This is well-defined by $[\mathbf{R}]$. Let $i \in \mathbb{I}$. We recall the following definitions:

$\diamond$ The reflection $s_{i}^{\mathfrak{q}} \in G L\left(\mathbb{Z}^{\theta}\right)$, given by $s_{i}^{\mathfrak{q}}\left(\alpha_{j}\right)=\alpha_{j}-c_{i j}^{\mathfrak{q}} \alpha_{i}, j \in \mathbb{I}$.

$\diamond$ The matrix $\rho_{i}(\mathfrak{q})$, given by $\rho_{i}(\mathfrak{q})_{j k}=\mathbf{q}\left(s_{i}^{\mathfrak{q}}\left(\alpha_{j}\right), s_{i}^{\mathfrak{q}}\left(\alpha_{k}\right)\right), j, k \in \mathbb{I}$.

$\diamond$ The braided vector space $\rho_{i}(V)$ of diagonal type with matrix $\rho_{i}(\mathfrak{q})$.

A basic result is that $\mathcal{B}_{\mathfrak{q}} \simeq \mathcal{B}_{\rho_{i}(\mathfrak{q})}$, at least as graded vector spaces.

The algebras $T(V)$ and $\mathcal{B}_{\mathfrak{q}}$ are $\mathbb{Z}^{\theta}$-graded by $\operatorname{deg} x_{i}=\alpha_{i}, i \in \mathbb{I}$. Let $\Delta_{+}^{\mathfrak{q}}$ be the set of $\mathbb{Z}^{\theta}$-degrees of the generators of a PBW-basis of $\mathcal{B}_{\mathfrak{q}}$, counted with multiplicities [H1]. The elements of $\Delta_{+}^{\mathfrak{q}}$ are called (positive) roots. Let $\Delta^{\mathfrak{q}}=\Delta_{+}^{\mathfrak{q}} \cup-\Delta_{+}^{\mathfrak{q}}$. Let

$$
\mathcal{X}:=\left\{\rho_{j_{1}} \ldots \rho_{j_{N}}(\mathfrak{q}): j_{1}, \ldots, j_{N} \in \mathbb{I}, N \in \mathbb{N}\right\} .
$$

Then the generalized root system of $\mathfrak{q}$ is the fibration $\Delta \rightarrow \mathcal{X}$, where the fiber of $\rho_{j_{1}} \ldots \rho_{j_{N}}(\mathfrak{q})$ is $\Delta^{\rho_{j_{1}} \ldots \rho_{j_{N}}(\mathfrak{q})}$. The Weyl groupoid of $\mathcal{B}_{\mathfrak{q}}$ is a groupoid, denoted $\mathcal{W}_{\mathfrak{q}}$, that acts on this fibration, generalizing the classical Weyl group, see [H1]. We know from loc. cit. that $\mathcal{W}_{\mathfrak{q}}$ is finite (and this characterizes finite-dimensional Nichols algebras of diagonal type).

Here is a useful description of $\Delta_{+}^{\mathfrak{q}}$. Let $w \in \mathcal{W}_{\mathfrak{q}}$ be an element of maximal length. We fix a reduced expression $w=\sigma_{i_{1}}^{\mathfrak{q}} \sigma_{i_{2}} \cdots \sigma_{i_{M}}$. For $1 \leq k \leq M$ set

$$
\beta_{k}=s_{i_{1}}^{\mathfrak{q}} \cdots s_{i_{k-1}}\left(\alpha_{i_{k}}\right),
$$

Then $\Delta_{+}^{\mathfrak{q}}=\left\{\beta_{k} \mid 1 \leq k \leq M\right\}\left[\mathrm{CH}\right.$, Prop. 2.12]; in particular $\left|\Delta_{+}^{\mathfrak{q}}\right|=M$.

The notion of Cartan root is instrumental for the definitions of $\widetilde{\mathcal{B}}_{\mathfrak{q}}$ and $\mathcal{L}_{\mathfrak{q}}$. First, following [A5] we say that $i \in \mathbb{I}$ is a Cartan vertex of $\mathfrak{q}$ if

$$
q_{i j} q_{j i}=q_{i i}^{c_{i j}^{\mathfrak{q}}}, \quad \text { for all } j \neq i,
$$

Then the set of Cartan roots of $\mathfrak{q}$ is

$$
\mathfrak{O}_{\mathfrak{q}}=\left\{s_{i_{1}}^{\mathfrak{q}} s_{i_{2}} \ldots s_{i_{k}}\left(\alpha_{i}\right) \in \Delta_{+}^{\mathfrak{q}}: i \in \mathbb{I} \text { is a Cartan vertex of } \rho_{i_{k}} \ldots \rho_{i_{2}} \rho_{i_{1}}(\mathfrak{q})\right\} \text {. }
$$


Given a positive root $\beta \in \Delta_{+}^{\mathfrak{q}}$, there is an associated root vector $x_{\beta} \in \mathcal{B}_{\mathfrak{q}}$ defined via the so-called Lusztig isomorphisms [H3]. Set $N_{\beta}=\operatorname{ord} q_{\beta \beta} \in \mathbb{N}$, $\beta \in \Delta_{+}^{\mathfrak{q}}$. Also, for $\mathbf{h}=\left(h_{1}, \ldots, h_{M}\right) \in \mathbb{N}_{0}^{M}$ we write

$$
x^{\mathbf{h}}=x_{\beta_{M}}^{h_{M}} x_{\beta_{M-1}}^{h_{M-1}} \cdots x_{\beta_{1}}^{h_{1}} .
$$

Let $\widetilde{N}_{k}= \begin{cases}N_{\beta_{k}} & \text { if } \beta_{k} \notin \mathcal{O}_{\mathfrak{q}}, \\ \infty & \text { if } \beta_{k} \in \mathcal{O}_{\mathfrak{q}} .\end{cases}$

$$
\mathrm{H}=\left\{\mathbf{h} \in \mathbb{N}_{0}^{M}: 0 \leq h_{k}<\widetilde{N}_{k} \text {, for all } k \in \mathbb{I}_{M}\right\} .
$$

By [A5, Theorem 3.6] the set $\left\{x^{\mathbf{h}} \mid \mathbf{h} \in \mathrm{H}\right\}$ is a basis of $\widetilde{\mathcal{B}}_{\mathfrak{q}}$.

As said in the Introduction, the Lusztig algebra associated to $\mathcal{B}_{\mathfrak{q}}$ is the braided Hopf algebra $\mathcal{L}_{\mathfrak{q}}$ which is the graded dual of $\widetilde{\mathcal{B}}_{\mathfrak{q}}$. Thus, it comes equipped with a bilinear form $\langle\rangle:, \widetilde{\mathcal{B}}_{\mathfrak{q}} \times \mathcal{L}_{\mathfrak{q}} \rightarrow \mathbf{k}$, which satisfies for all $x, x^{\prime} \in \widetilde{\mathcal{B}}_{\mathfrak{q}}, y, y^{\prime} \in \mathcal{L}_{\mathfrak{q}}$

$$
\left\langle y, x x^{\prime}\right\rangle=\left\langle y^{(2)}, x\right\rangle\left\langle y^{(1)}, x^{\prime}\right\rangle \quad \text { and } \quad\left\langle y y^{\prime}, x\right\rangle=\left\langle y, x^{(2)}\right\rangle\left\langle y^{\prime}, x^{(1)}\right\rangle .
$$

If $\mathbf{h} \in \mathrm{H}$, then define $\mathbf{y}_{\mathbf{h}} \in \mathcal{L}_{\mathfrak{q}}$ by $\left\langle\mathbf{y}_{\mathbf{h}}, x^{\mathbf{j}}\right\rangle=\delta_{\mathbf{h}, \mathbf{j}}, \mathbf{j} \in \mathrm{H}$. Let $\left(\mathbf{h}_{k}\right)_{k \in \mathbb{I}_{M}}$ denote the canonical basis of $\mathbb{Z}^{M}$. If $k \in \mathbb{I}_{M}$ and $\beta=\beta_{k} \in \Delta_{+}^{\mathfrak{q}}$, then we denote the element $\mathbf{y}_{n \mathbf{h}_{k}}$ by $y_{\beta}^{(n)}$. Then the algebra $\mathcal{L}_{\mathfrak{q}}$ is generated by

$$
\left\{y_{\alpha}: \alpha \in \Pi_{\mathfrak{q}}\right\} \cup\left\{y_{\alpha}^{\left(N_{\alpha}\right)}: \alpha \in \mathfrak{O}_{\mathfrak{q}}, x_{\alpha}^{N_{\alpha}} \in \mathcal{P}\left(\widetilde{\mathcal{B}}_{\mathfrak{q}}\right)\right\},
$$

by AAR]. Moreover, by [AAR, 4.6], the following set is a basis of $\mathcal{L}_{\mathfrak{q}}$ :

$$
\left\{y_{\beta_{1}}^{\left(h_{1}\right)} \cdots y_{\beta_{M}}^{\left(h_{M}\right)} \mid\left(h_{1}, \ldots, h_{M}\right) \in \mathrm{H}\right\} \text {. }
$$

2.2. Lyndon words, convex order and PBW-basis. For the computations in Section 4 we need some preliminaries on Kharchenko's PBWbasis. Let $(V, \mathfrak{q})$ be as above and let $\mathbb{X}$ be the set of words with letters in $X=\left\{x_{1}, \ldots, x_{\theta}\right\}$ (our fixed basis of $V$ ); the empty word is 1 and for $u \in \mathbb{X}$ we write $\ell(u)$ the length of $u$. We can identify $\mathbf{k} \mathbb{X}$ with $T(V)$.

Definition 2.1. Consider the lexicographic order in $\mathbb{X}$. We say that $u \in$ $\mathbb{X}-\{1\}$ is a Lyndon word if for every decomposition $u=v w, v, w \in \mathbb{X}-\{1\}$, then $u<w$. We denote by $L$ the set of all Lyndon words.

A well-known theorem, due to Lyndon, established that any word $u \in \mathbb{X}$ admits a unique decomposition, named Lyndon decomposition, as a nonincreasing product of Lyndon words:

$$
u=l_{1} l_{2} \ldots l_{r}, \quad l_{i} \in L, l_{r} \leq \cdots \leq l_{1} .
$$

Also, each $l_{i} \in L$ in (7) is called a Lyndon letter of $u$.

Now each $u \in L-X$ admits at least one decomposition $u=v_{1} v_{2}$ with $v_{1}, v_{2} \in L$. Then the Shirshov decomposition of $u$ is the decomposition $u=u_{1} u_{2}, u_{1}, u_{2} \in L$, such that $u_{2}$ is the smallest end of $u$ between all possible decompositions of this form. 
For any braided vector space $V$, the braided bracket of $x, y \in T(V)$ is

$$
[x, y]_{c}:=\text { multiplication } \circ(\mathrm{id}-c)(x \otimes y) .
$$

Using the identification $T(V)=\mathbf{k} \mathbb{X}$ and the decompositions described above, we can define a k-linear endomorphism $[-]_{c}$ of $T(V)$ as follows:

$$
[u]_{c}:= \begin{cases}u, & \text { if } u=1 \text { or } u \in X ; \\ {\left[[v]_{c},[w]_{c}\right]_{c},} & \text { if } u \in L-X, u=v w \text { its Shirshov decomposition; } \\ {\left[u_{1}\right]_{c} \ldots\left[u_{t}\right]_{c},} & \text { if } u \in \mathbb{X}-L, u=u_{1} \ldots u_{t} \text { its Lyndon decomposition. }\end{cases}
$$

We will describe PBW-bases using this endomorphism.

Definition 2.2. For $l \in L$, the element $[l]_{c}$ is the corresponding hyperletter. A word written in hyperletters is an hyperword; a monotone hyperword is an hyperword $W=\left[u_{1}\right]_{c}^{k_{1}} \ldots\left[u_{m}\right]_{c}^{k_{m}}$ such that $u_{1}>\cdots>u_{m}$.

Consider now a different order on $\mathbb{X}$, called deg-lex order $[\mathrm{K}$ : For each pair $u, v \in \mathbb{X}$, we have that $u \succ v$ if $\ell(u)<\ell(v)$, or $\ell(u)=\ell(v)$ and $u>v$ for the lexicographical order. This order is total, the empty word 1 is the maximal element and it is invariant by left and right multiplication.

Let $I$ be a Hopf ideal of $T(V)$ and $R=T(V) / I$. Let $\pi: T(V) \rightarrow R$ be the canonical projection. We set:

$$
G_{I}:=\left\{u \in \mathbb{X}: u \notin \mathbf{k} \mathbb{X}_{\succ u}+I\right\} .
$$

Thus, if $u \in G_{I}$ and $u=v w$, then $v, w \in G_{I}$. So, each $u \in G_{I}$ is a non-increasing product of Lyndon words of $G_{I}$.

Let $S_{I}:=G_{I} \cap L$ and let $h_{I}: S_{I} \rightarrow\{2,3, \ldots\} \cup\{\infty\}$ be defined by:

$$
h_{I}(u):=\min \left\{t \in \mathbb{N}: u^{t} \in \mathbf{k} \mathbb{X}_{\succ u^{t}}+I\right\} .
$$

Theorem 2.3. $\mathrm{K}$ The following set is a $P B W$-basis of $R=T(V) / I$ :

$$
\left\{\left[u_{1}\right]_{c}^{k_{1}} \ldots\left[u_{m}\right]_{c}^{k_{m}}: m \in \mathbb{N}_{0}, u_{1}>\ldots>u_{m}, u_{i} \in S_{I}, 0<k_{i}<h_{I}\left(u_{i}\right)\right\} .
$$

We refer to this base as Kharchenko's PBW-basis of $T(V) / I$ (it depends on the order of $X$ ).

Definition 2.4. [A2, 2.6] Let $\Delta_{\mathfrak{q}}^{+}$be as above and let $<$be a total order on $\Delta_{\mathfrak{q}}^{+}$. We say that the order is convex if for each $\alpha, \beta \in \Delta_{\mathfrak{q}}^{+}$such that $\alpha<\beta$ and $\alpha+\beta \in \Delta_{\mathfrak{q}}^{+}$, then $\alpha<\alpha+\beta<\beta$. The order is called strongly convex if for each ordered subset $\alpha_{1} \leq \alpha_{2} \leq \cdots \leq \alpha_{k}$ of elements of $\Delta_{\mathfrak{q}}^{+}$such that $\alpha=\sum_{i} \alpha_{i} \in \Delta_{\mathfrak{q}}^{+}$, then $\alpha_{1}<\alpha<\alpha_{k}$.

Theorem 2.5. [A2, 2.11] The following statements are equivalent:

- The order is convex.

- The order is strongly convex.

- The order arises from a reduced expression of a longest element $w \in \mathcal{W}_{\mathfrak{q}}$, cf. (44). 
Now, we have two PBW-basis of $\mathcal{B}_{q}$ (and correspondingly of $\widetilde{\mathcal{B}}_{\mathfrak{q}}$ ), namely Kharchenko's PBW-basis and the PBW-basis defined from a reduced expression of a longest element of the Weyl groupoid. But both basis are reconciled by [AY, Theorem 4.12], thanks to [A2, 2.14]. Indeed, each generator of Kharchenko's PBW-basis is a multiple scalar of a generator of the secondly mentioned PBW-basis. So, for ease of calculations, in the rest of this work we shall use the Kharchenko generators.

The following proposition is used to compute the hyperword $\left[l_{\beta}\right]_{c}$ associated to a $\operatorname{root} \beta \in \Delta_{\mathfrak{q}}^{+}$:

Proposition 2.6. [A2, 2.17] For $\beta \in \Delta_{\mathfrak{q}}^{+}$,

$l_{\beta}= \begin{cases}x_{\alpha_{i}}, & \text { if } \beta=\alpha_{i}, i \in \mathbb{I} \\ \max \left\{l_{\delta_{1}} l_{\delta_{2}}: \delta_{1}, \delta_{2} \in \Delta_{\mathfrak{q}}^{+}, \delta_{1}+\delta_{2}=\beta, l_{\delta_{1}}<l_{\delta_{2}}\right\}, & \text { if } \beta \neq \alpha_{i}, i \in \mathbb{I} .\end{cases}$

We give a list of the hyperwords appearing in the next section:

\begin{tabular}{ccc} 
Root & Hyperword & Notation \\
\hline$\alpha_{i}$ & $x_{i}$ & $x_{i}$ \\
$n \alpha_{1}+\alpha_{2}$ & $\left(\operatorname{ad}_{c} x_{1}\right)^{n} x_{2}$ & $x_{1 \ldots 12}$ \\
$\alpha_{1}+2 \alpha_{2}$ & {$\left[x_{\alpha_{1}+\alpha_{2}}, x_{2}\right]_{c}$} & {$\left[x_{12}, x_{2}\right]_{c}$} \\
$3 \alpha_{1}+2 \alpha_{2}$ & {$\left[x_{2 \alpha_{1}+\alpha_{2}}, x_{\alpha_{1}+\alpha_{2}}\right]_{c}$} & {$\left[x_{112}, x_{12}\right]_{c}$} \\
$4 \alpha_{1}+3 \alpha_{2}$ & {$\left[x_{3 \alpha_{1}+2 \alpha_{2}}, x_{\alpha_{1}+\alpha_{2}}\right]_{c}$} & {$\left[\left[x_{112}, x_{12}\right]_{c}, x_{12}\right]_{c}$} \\
$5 \alpha_{1}+3 \alpha_{2}$ & {$\left[x_{2 \alpha_{1}+\alpha_{2}}, x_{3 \alpha_{1}+2 \alpha_{2}}\right]_{c}$} & {$\left[x_{112},\left[x_{112}, x_{12}\right]_{c}\right]_{c}$}
\end{tabular}

We use an analogous notation for the elements of $\mathcal{L}_{\mathfrak{q}}$ : for example we write $y_{112,12}$ when we refer to the element of $\mathcal{L}_{\mathfrak{q}}$ which corresponds to $\left[x_{112}, x_{12}\right]_{c}$.

\section{Extensions of BRAided Hopf Algebras}

We recall the definition of braided Hopf algebra extensions given in [AN]; we refer to $\mathrm{BD}, \mathrm{GG}$, for more general definitions. Below we denote by $\Delta$ the coproduct of a braided Hopf algebra $A$ and by $A^{+}$the kernel of the counit.

First, if $\pi: C \rightarrow B$ is a morphism of Hopf algebras in ${ }_{H}^{H} \mathcal{Y} \mathcal{D}$, then we set

$$
\begin{aligned}
& C^{\text {co } \pi}=\{c \in C \mid(\mathrm{id} \otimes \pi) \underline{\Delta}(c)=c \otimes 1\}, \\
& { }^{\text {co } \pi} C=\{c \in C \mid(\pi \otimes \mathrm{id}) \underline{\Delta}(c)=1 \otimes c\} .
\end{aligned}
$$

Definition 3.1. AN, $\S 2.5]$ Let $H$ be a Hopf algebra. A sequence of morphisms of Hopf algebras in ${ }_{H}^{H} \mathcal{Y} \mathcal{D}$

$$
\mathbf{k} \rightarrow A \stackrel{\iota}{\rightarrow} C \stackrel{\pi}{\rightarrow} B \rightarrow \mathbf{k}
$$

is an extension of braided Hopf algebras if

(i) $\iota$ is injective,

(ii) $\pi$ is surjective,

(iii) $\operatorname{ker} \pi=C \iota\left(A^{+}\right)$and

(iv) $A=C^{\text {co } \pi}$, or equivalently $A={ }^{\operatorname{co} \pi} C$.

For simplicity, we shall write $A \stackrel{\iota}{\hookrightarrow} C \stackrel{\pi}{\rightarrow} B$ instead of (10). 
This Definition applies in our context: recall that $\mathcal{B}_{\mathfrak{q}} \simeq \widetilde{\mathcal{B}}_{\mathfrak{q}} /\left\langle x_{\beta}^{N_{\beta}}, \beta \in \mathfrak{O}_{\mathfrak{q}}\right\rangle$. Let $Z_{\mathfrak{q}}$ be the subalgebra of $\widetilde{\mathcal{B}}_{\mathfrak{q}}$ generated by $x_{\beta}^{N_{\beta}}, \beta \in \mathfrak{O}_{\mathfrak{q}}$. Then

$\circ$ The inclusion $\iota: Z_{\mathfrak{q}} \rightarrow \widetilde{\mathcal{B}}_{\mathfrak{q}}$ is injective and the projection $\pi: \widetilde{\mathcal{B}}_{\mathfrak{q}} \rightarrow \mathcal{B}_{\mathfrak{q}}$ is surjective.

$\circ$ [A5, Theorem 4.10] $Z_{\mathfrak{q}}$ is a normal Hopf subalgebra of $\widetilde{\mathcal{B}}_{\mathfrak{q}}$; since ker $\pi$ is the two-sided ideal generated by $\iota\left(Z_{\mathfrak{q}}^{+}\right)$, $\operatorname{ker} \pi=\widetilde{\mathcal{B}}_{\mathfrak{q}} \iota\left(Z_{\mathfrak{q}}^{+}\right)$.

$\circ$ [A5, Theorem 4.13] $Z_{\mathfrak{q}}={ }^{\operatorname{co} \pi} \widetilde{\mathcal{B}}_{\mathfrak{q}}$.

Hence we have an extension of braided Hopf algebras

$$
Z_{\mathfrak{q}} \stackrel{\iota}{\hookrightarrow} \widetilde{\mathcal{B}}_{\mathfrak{q}} \stackrel{\pi}{\rightarrow} \mathcal{B}_{\mathfrak{q}} .
$$

The morphisms $\iota$ and $\pi$ are graded. Thus, taking graded duals, we obtain a new sequence of morphisms of braided Hopf algebras

$$
\mathcal{B}_{\mathfrak{q}} \stackrel{\pi^{*}}{\rightarrow} \mathcal{L}_{\mathfrak{q}} \stackrel{\iota^{*}}{\rightarrow} \mathfrak{Z}_{\mathfrak{q}}
$$

Proposition 3.2. The sequence (2) is an extension of braided Hopf algebras.

Proof. The argument of [A, 3.3.1] can be adapted to the present situation, or more generally to extensions of braided Hopf algebras that are graded with finite-dimensional homogeneous components. The map $\pi^{*}: \mathcal{B}_{\mathfrak{q}} \rightarrow \mathcal{L}_{\mathfrak{q}}$ is injective because $\mathcal{B}_{\mathfrak{q}} \simeq \mathcal{B}_{\mathfrak{q}}^{*} ; \iota^{*}: \mathcal{L}_{\mathfrak{q}} \stackrel{\iota^{*}}{\rightarrow} \mathfrak{Z}_{\mathfrak{q}}$ is surjective being the transpose of a graded monomorphism between two locally finite graded vector spaces. Now, since $Z_{\mathfrak{q}}={ }^{\operatorname{co} \pi} \widetilde{\mathcal{B}}_{\mathfrak{q}}=\widetilde{\mathcal{B}}_{\mathfrak{q}}^{\text {co } \pi}$, we have

$$
\operatorname{ker} \iota^{*}=\mathcal{L}_{\mathfrak{q}} \mathcal{B}_{\mathfrak{q}}^{+}=\mathcal{B}_{\mathfrak{q}}^{+} \mathcal{L}_{\mathfrak{q}} \text {. }
$$

Similarly $\mathcal{L}_{\mathfrak{q}}^{\text {co } \iota^{*}}=\mathcal{B}_{\mathfrak{q}}^{*}$ because $\operatorname{ker} \pi^{\perp}=\mathcal{B}_{\mathfrak{q}}$.

From now on, we assume the condition (11) on the matrix $\mathfrak{q}$ mentioned in the Introduction, that is

$$
q_{\alpha \beta}^{N_{\beta}}=1, \quad \forall \alpha, \beta \in \mathfrak{O}_{\mathfrak{q}} .
$$

The following result is our basic tool to compute the Lie algebra $\mathfrak{n}_{\mathfrak{q}}$.

Theorem 3.3. The braided Hopf algebra $\mathfrak{Z}_{\mathfrak{q}}$ is an usual Hopf algebra, isomorphic to the universal enveloping algebra of the Lie algebra $\mathfrak{n}_{\mathfrak{q}}=\mathcal{P}\left(\mathfrak{Z}_{\mathfrak{q}}\right)$. The elements $\xi_{\beta}:=\iota^{*}\left(y_{\beta}^{\left(N_{\beta}\right)}\right), \beta \in \mathfrak{O}_{\mathfrak{q}}$, form a basis of $\mathfrak{n}_{\mathfrak{q}}$.

Proof. Let $A_{\mathfrak{q}}$ be the subspace of $\mathcal{L}_{\mathfrak{q}}$ generated by the ordered monomials

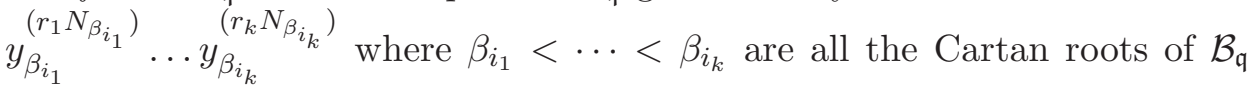
and $r_{1}, \ldots, r_{k} \in \mathbb{N}_{0}$. We claim that the restriction of the multiplication $\mu: \mathcal{B}_{\mathfrak{q}} \otimes A_{\mathfrak{q}} \rightarrow \mathcal{L}_{\mathfrak{q}}$ is an isomorphism of vector spaces. Indeed, $\mu$ is surjective 
by the commuting relations in $\mathcal{L}_{\mathfrak{q}}$. Also, the Hilbert series of $\mathcal{L}_{\mathfrak{q}}, \mathcal{B}_{\mathfrak{q}}$ and $A_{\mathfrak{q}}$ are respectively:

$$
\begin{aligned}
& \mathcal{H}_{\mathcal{L}_{\mathfrak{q}}}=\prod_{\beta_{k} \in \mathfrak{O}_{\mathfrak{q}}} \frac{1}{1-T^{\operatorname{deg} \beta}} \cdot \prod_{\beta_{k} \notin \mathfrak{O}_{\mathfrak{q}}} \frac{1-T^{N_{\beta} \operatorname{deg} \beta}}{1-T^{\operatorname{deg} \beta}} ; \\
& \mathcal{H}_{\mathcal{B}_{\mathfrak{q}}}=\prod_{\beta_{k} \in \Delta_{\mathfrak{q}}^{+}} \frac{1-T^{N_{\beta} \operatorname{deg} \beta}}{1-T^{\operatorname{deg} \beta}} \\
& \mathcal{H}_{A_{\mathfrak{q}}}=\prod_{\beta_{k} \in \mathfrak{O}_{\mathfrak{q}}} \frac{1}{1-T^{N_{\beta} \operatorname{deg} \beta}} .
\end{aligned}
$$

Since the multiplication is graded and $\mathcal{H}_{\mathcal{L}_{\mathfrak{q}}}=\mathcal{H}_{\mathcal{B}_{\mathfrak{q}}} \mathcal{H}_{A_{\mathfrak{q}}}, \mu$ is injective. The claim follows and we have

$$
\mathcal{L}_{\mathfrak{q}}=A_{\mathfrak{q}} \oplus \mathcal{B}_{\mathfrak{q}}^{+} A_{\mathfrak{q}}
$$

We next claim that $\iota^{*}: A_{\mathfrak{q}} \rightarrow \mathfrak{Z}_{\mathfrak{q}}$ is an isomorphism of vector spaces. Indeed, by (12), $\operatorname{ker} \iota^{*}=\mathcal{B}_{\mathfrak{q}}^{+} \mathcal{L}_{\mathfrak{q}}=\mathcal{B}_{\mathfrak{q}}^{+}\left(\mathcal{B}_{\mathfrak{q}} A_{\mathfrak{q}}\right)=\mathcal{B}_{\mathfrak{q}}^{+} A_{\mathfrak{q}}$. By (13), the claim follows.

By (1), $Z_{\mathfrak{q}}$ is a commutative Hopf algebra, see [A5; hence $\mathfrak{Z}_{\mathfrak{q}}$ is a cocommutative Hopf algebra. Now the elements $\xi_{\beta}:=\iota^{*}\left(y_{\beta}^{\left(N_{\beta}\right)}\right), \beta \in \mathfrak{O}_{\mathfrak{q}}$, are primitive, i.e. belong to $\mathfrak{n}_{\mathfrak{q}}=\mathcal{P}\left(\mathfrak{Z}_{\mathfrak{q}}\right)$. The monomials $\xi_{\beta_{i_{1}}}^{r_{1}} \ldots \xi_{\beta_{i_{k}}}^{r_{k}}$, $\beta_{i_{1}}<\cdots<\beta_{i_{k}} \in \mathfrak{O}_{\mathfrak{q}}, r_{1}, \ldots, r_{k} \in \mathbb{N}_{0}$ form a basis of $\mathfrak{Z}_{\mathfrak{q}}$, hence

$$
\mathfrak{Z}_{\mathfrak{q}}=\mathbf{k}\left\langle\xi_{\beta}: \beta \in \mathfrak{O}_{\mathfrak{q}}\right\rangle \subseteq \mathcal{U}\left(\mathfrak{n}_{\mathfrak{q}}\right) \subseteq \mathfrak{Z}_{\mathfrak{q}} .
$$

We conclude that $\left(\xi_{\beta}\right)_{\beta \in \mathcal{O}_{\mathfrak{q}}}$ is a basis of $\mathfrak{n}_{\mathfrak{q}}$ and that $\mathfrak{Z}_{\mathfrak{q}}=\mathcal{U}\left(\mathfrak{n}_{\mathfrak{q}}\right)$.

\section{Proof of Theorem 1.1}

In this section we consider all indecomposable matrices $\mathfrak{q}$ of rank 2 whose associated Nichols algebra $\mathcal{B}_{\mathfrak{q}}$ is finite-dimensional; these are classified in [H2 and we recall their diagrams in Table 1. For each $\mathfrak{q}$ we obtain an isomorphism between $\mathfrak{Z}_{\mathfrak{q}}$ and $\mathcal{U}\left(\mathfrak{g}^{+}\right)$, the universal enveloping algebra of the positive part of $\mathfrak{g}$. Here $\mathfrak{g}$ is the semisimple Lie algebra of the last column of Table 1, with Cartan matrix $A=\left(a_{i j}\right)_{1 \leq i, j \leq 2}$. By simplicity we denote $\mathfrak{g}$ by its type, e.g. $\mathfrak{g}=A_{2}$.

We recall that we assume (11) and that $\xi_{\beta}=\iota^{*}\left(y_{\beta}^{\left(N_{\beta}\right)}\right) \in \mathfrak{Z}_{\mathfrak{q}}$. Thus,

$$
\left[\xi_{\alpha}, \xi_{\beta}\right]_{c}=\xi_{\alpha} \xi_{\beta}-\xi_{\beta} \xi_{\alpha}=\left[\xi_{\alpha}, \xi_{\beta}\right], \quad \text { for all } \alpha, \beta \in \mathfrak{O}_{\mathfrak{q}} .
$$

The strategy to prove the isomorphism $\mathfrak{F}: \mathcal{U}\left(\mathfrak{g}^{+}\right) \rightarrow \mathfrak{Z}_{\mathfrak{q}}$ is the following:

(1) If $\mathfrak{O}_{\mathfrak{q}}=\emptyset$, then $\mathfrak{g}^{+}=0$. If $\left|\mathfrak{O}_{\mathfrak{q}}\right|=1$, then $\mathfrak{g}=\mathfrak{s l}_{2}$, i.e. of type $A_{1}$.

(2) If $\left|\mathfrak{O}_{\mathfrak{q}}\right|=2$, then $\mathfrak{g}$ is of type $A_{1} \oplus A_{1}$. Indeed, let $\mathfrak{O}_{\mathfrak{q}}=\{\alpha, \beta\}$. As $\mathfrak{Z}_{\mathfrak{q}}$ is $\mathbb{N}_{0}^{\theta}$-graded, $\left[\xi_{\alpha}, \xi_{\beta}\right] \in \mathfrak{n}_{\mathfrak{q}}$ has degree $N_{\alpha} \alpha+N_{\beta} \beta$. Thus $\left[\xi_{\alpha}, \xi_{\beta}\right]=0$.

(3) Now assume that $\left|\mathfrak{O}_{\mathfrak{q}}\right|>2$. We recall that $\mathfrak{Z}_{\mathfrak{q}}$ is generated by

$$
\left\{\xi_{\beta} \mid x_{\beta}^{N_{\beta}} \text { is a primitive element of } \widetilde{\mathcal{B}}_{\mathfrak{q}}\right\} \text {. }
$$


We compute the coproduct of all $x_{\beta}^{N_{\beta}}$ in $\widetilde{\mathcal{B}}_{\mathfrak{q}}, \beta \in \mathfrak{O}_{\mathfrak{q}}$, using that $\triangleq$ is a graded map and $Z_{\mathfrak{q}}$ is a Hopf subalgebra of $\widetilde{\mathcal{B}}_{\mathfrak{q}}$. In all cases we get two primitive elements $x_{\beta_{1}}^{N_{\beta_{1}}}$ and $x_{\beta_{2}}^{N_{\beta_{2}}}$, thus $\mathfrak{Z}_{\mathfrak{q}}$ is generated by $\xi_{\beta_{1}}$ and $\xi_{\beta_{2}}$.

(4) Using the coproduct again, we check that

$$
\left(\operatorname{ad} \xi_{\beta_{i}}\right)^{1-a_{i j}} \xi_{\beta_{j}}=0, \quad 1 \leq i \neq j \leq 2 .
$$

To prove (14), it is enough to observe that $\mathfrak{n}_{\mathfrak{q}}$ has a trivial component of degree $N_{\beta_{i}}\left(1-a_{i j}\right) \beta_{i}+N_{\beta_{j}} \beta_{j}$. Now (14) implies that there exists a surjective map of Hopf algebras $\mathfrak{F}: \mathcal{U}\left(\mathfrak{g}^{+}\right) \rightarrow \mathfrak{Z}_{\mathfrak{q}}$ such that $e_{i} \mapsto \xi_{\beta_{i}}$.

(5) To prove that $\mathfrak{F}$ is an isomorphism, it suffices to see that the restriction $\mathfrak{g}^{+} \stackrel{*}{\rightarrow} \mathfrak{n}_{\mathfrak{q}}$ is an isomorphism; but in each case we see that $*$ is surjective, and $\operatorname{dim} \mathfrak{g}^{+}=\operatorname{dim} \mathfrak{n}_{\mathfrak{q}}=\left|\mathfrak{O}_{\mathfrak{q}}\right|$.

We refer to [A1, AAY, A4] for the presentation, root system and Cartan roots of braidings of standard, super and unidentified type respectively.

Row 1. Let $q \in \mathbb{G}_{N}^{\prime}, N \geq 2$. The diagram $\stackrel{q}{\circ} q^{-1}{ }^{q}$ corresponds to a braiding of Cartan type $A_{2}$ whose set of positive roots is $\Delta_{\mathfrak{q}}^{+}=\left\{\alpha_{1}, \alpha_{1}+\right.$ $\left.\alpha_{2}, \alpha_{2}\right\}$. In this case $\mathfrak{O}_{\mathfrak{q}}=\Delta_{\mathfrak{q}}^{+}$and $N_{\beta}=N$ for all $\beta \in \mathfrak{O}_{\mathfrak{q}}$. By hypothesis, $q_{12}^{N}=q_{21}^{N}=1$. The elements $x_{1}, x_{2} \in \widetilde{\mathcal{B}}_{\mathfrak{q}}$ are primitive and

$$
\triangleq\left(x_{12}\right)=x_{12} \otimes 1+1 \otimes x_{12}+\left(1-q^{-1}\right) x_{1} \otimes x_{2} .
$$

Then the coproducts of the elements $x_{1}^{N}, x_{12}^{N}, x_{2}^{N} \in \widetilde{\mathcal{B}}_{\mathfrak{q}}$ are:

$$
\begin{aligned}
& \underline{\Delta}\left(x_{1}^{N}\right)=x_{1}^{N} \otimes 1+1 \otimes x_{1}^{N} ; \quad \underline{\Delta}\left(x_{2}^{N}\right)=x_{2}^{N} \otimes 1+1 \otimes x_{2}^{N} ; \\
& \underline{\Delta}\left(x_{12}^{N}\right)=x_{12}^{N} \otimes 1+1 \otimes x_{12}^{N}+\left(1-q^{-1}\right)^{N} q_{21}^{\frac{N(N-1)}{2}} x_{1}^{N} \otimes x_{2}^{N} .
\end{aligned}
$$

As $\left[\xi_{2}, \xi_{12}\right],\left[\xi_{1}, \xi_{12}\right] \in \mathfrak{n}_{\mathfrak{q}}$ have degree $N \alpha_{1}+2 N \alpha_{2}$, respectively $2 N \alpha_{1}+N \alpha_{2}$, and the components of these degrees of $\mathfrak{n}_{\mathfrak{q}}$ are trivial, we have

$$
\left[\xi_{2}, \xi_{12}\right]=\left[\xi_{1}, \xi_{12}\right]=0
$$

Again by degree considerations, there exists $c \in \mathbf{k}$ such that $\left[\xi_{2}, \xi_{1}\right]=c \xi_{12}$. By the duality between $\mathfrak{Z}_{\mathfrak{q}}$ and $Z_{\mathfrak{q}}$ we have that

$$
\left[\xi_{2}, \xi_{1}\right]=\left(1-q^{-1}\right)^{N} q_{21}^{\frac{N(N-1)}{2}} \xi_{12} .
$$

Then there exists a morphism of algebras $\mathfrak{F}: \mathcal{U}\left(A_{2}^{+}\right) \rightarrow \mathfrak{Z}_{\mathfrak{q}}$ given by

$$
e_{1} \mapsto \xi_{1}, \quad e_{2} \mapsto \xi_{2} .
$$

This morphism takes a basis of $A_{2}^{+}$to a basis of $\mathfrak{n}_{\mathfrak{q}}$, so $\mathfrak{Z}_{\mathfrak{q}} \simeq \mathcal{U}\left(A_{2}^{+}\right)$.

Row 2. Let $q \in \mathbb{G}_{N}^{\prime}, N \geq 3$. These diagrams correspond to braidings of super type $A$ with positive roots $\Delta_{\mathfrak{q}}^{+}=\left\{\alpha_{1}, \alpha_{1}+\alpha_{2}, \alpha_{2}\right\}$. 
The first diagram is $\stackrel{q}{q} q^{-1}-1$. In this case the unique Cartan root is $\alpha_{1}$ with $N_{\alpha_{1}}=N$. The element $x_{1}^{N} \in \widetilde{\mathcal{B}}_{\mathfrak{q}}$ is primitive and $\mathfrak{Z}_{\mathfrak{q}}$ is generated by $\xi_{1}$. Hence $\mathfrak{Z}_{\mathfrak{q}} \simeq \mathcal{U}\left(A_{1}^{+}\right)$.

The second diagram gives a similar situation, since $\mathfrak{O}_{\mathfrak{q}}=\left\{\alpha_{1}+\alpha_{2}\right\}$.

Row 3. Let $q \in \mathbb{G}_{N}^{\prime}, N \geq 3$. The diagram $\stackrel{q}{\circ} q^{-2} q^{2}$ corresponds to a braiding of Cartan type $B_{2}$ with $\Delta_{\mathfrak{q}}^{+}=\left\{\alpha_{1}, 2 \alpha_{1}+\alpha_{2}, \alpha_{1}+\alpha_{2}, \alpha_{2}\right\}$. In this case $\mathfrak{O}_{\mathfrak{q}}=\Delta_{\mathfrak{q}}^{+}$. The coproducts of the generators of $\widetilde{\mathcal{B}}_{\mathfrak{q}}$ are:

$$
\begin{aligned}
\underline{\Delta}\left(x_{1}\right)= & x_{1} \otimes 1+1 \otimes x_{1} ; \quad \underline{\Delta}\left(x_{2}\right)=x_{2} \otimes 1+1 \otimes x_{2} ; \\
\underline{\Delta}\left(x_{12}\right)= & x_{12} \otimes 1+1 \otimes x_{12}+\left(1-q^{-2}\right) x_{1} \otimes x_{2} ; \\
\underline{\Delta}\left(x_{112}\right)= & x_{112} \otimes 1+1 \otimes x_{112}+\left(1-q^{-1}\right)\left(1-q^{-2}\right) x_{1}^{2} \otimes x_{2} \\
& +q\left(1-q^{-2}\right) x_{1} \otimes x_{12} .
\end{aligned}
$$

We have two different cases depending on the parity of $N$.

(1) If $N$ is odd, then $N_{\beta}=N$ for all $\beta \in \Delta_{\mathfrak{q}}^{+}$. In this case,

$$
\begin{aligned}
\underline{\Delta}\left(x_{1}^{N}\right)= & x_{1}^{N} \otimes 1+1 \otimes x_{1}^{N} ; \quad \underline{\Delta}\left(x_{2}^{N}\right)=x_{2}^{N} \otimes 1+1 \otimes x_{2}^{N} ; \\
\underline{\Delta}\left(x_{12}^{N}\right)= & x_{12}^{N} \otimes 1+1 \otimes x_{12}^{N}+\left(1-q^{-2}\right)^{N} x_{1}^{N} \otimes x_{2}^{N} ; \\
\underline{\Delta}\left(x_{112}^{N}\right)= & x_{112}^{N} \otimes 1+1 \otimes x_{112}^{N}+\left(1-q^{-1}\right)^{N}\left(1-q^{-2}\right)^{N} x_{1}^{2 N} \otimes x_{2}^{N} \\
& +C x_{1}^{N} \otimes x_{12}^{N},
\end{aligned}
$$

for some $C \in \mathbf{k}$. Hence, in $\mathfrak{Z}_{\mathfrak{q}}$ we have the relations

$$
\begin{aligned}
& {\left[\xi_{1}, \xi_{2}\right]=\left(1-q^{-2}\right)^{N} \xi_{12} ;} \\
& {\left[\xi_{12}, \xi_{1}\right]=C \xi_{112} ;} \\
& {\left[\xi_{1}, \xi_{2}\right]_{c}=\left(1-q^{-1}\right)^{N}\left(1-q^{-2}\right)^{N} \xi_{112}+\left(1-q^{-2}\right)^{N} \xi_{1} \xi_{12} ;} \\
& {\left[\xi_{1}, \xi_{112}\right]=\left[\xi_{2}, \xi_{12}\right]=0 .}
\end{aligned}
$$

Thus there exists an algebra map $\mathfrak{F}: \mathcal{U}\left(B_{2}^{+}\right) \rightarrow \mathfrak{Z}_{\mathfrak{q}}$ given by $e_{1} \mapsto \xi_{1}, e_{2} \mapsto \xi_{2}$. Moreover, $\mathfrak{F}$ is an isomorphism, and so $\mathfrak{Z}_{\mathfrak{q}} \simeq \mathcal{U}\left(B_{2}^{+}\right)$. Using the relations of $\mathcal{U}\left(B_{2}^{+}\right)$we check that $C=2\left(1-q^{-1}\right)^{N}\left(1-q^{-2}\right)^{N}$.

(2) If $N=2 M>2$, then $N_{\alpha_{1}}=N_{\alpha_{1}+\alpha_{2}}=N$ and $N_{2 \alpha_{1}+\alpha_{2}}=N_{\alpha_{2}}=M$.

In this case we have

$$
\begin{aligned}
& \underline{\Delta}\left(x_{1}^{N}\right)=x_{1}^{N} \otimes 1+1 \otimes x_{1}^{N} ; \quad \underline{\Delta}\left(x_{2}^{M}\right)=x_{2}^{M} \otimes 1+1 \otimes x_{2}^{M} ; \\
& \triangleq\left(x_{12}^{N}\right)=x_{12}^{N} \otimes 1+1 \otimes x_{12}^{N}+\left(1-q^{-2}\right)^{N} q_{21}^{M(N-1)} x_{1}^{N} \otimes x_{2}^{2 M} \\
& +\left(1-q^{-2}\right)^{M} q_{21}^{M^{2}} x_{112}^{M} \otimes x_{2}^{M} \text {; } \\
& \underline{\Delta}\left(x_{112}^{M}\right)=x_{112}^{M} \otimes 1+1 \otimes x_{112}^{M}+\left(1-q^{-1}\right)^{M}\left(1-q^{-2}\right)^{M} q_{21}^{M(M-1)} x_{1}^{N} \otimes x_{2}^{M} \text {. }
\end{aligned}
$$


Hence, the following relations hold in $\mathfrak{Z}_{\mathfrak{q}}$ :

$$
\begin{aligned}
& {\left[\xi_{2}, \xi_{1}\right]=\left(1-q^{-1}\right)^{M}\left(1-q^{-2}\right)^{M} q_{21}^{M(M-1)} \xi_{112} ;} \\
& {\left[\xi_{112}, \xi_{2}\right]=\left(1-q^{-2}\right)^{M} q_{21}^{M^{2}} \xi_{12} ;} \\
& {\left[\xi_{1}, \xi_{112}\right]=\left[\xi_{2}, \xi_{12}\right]=0 .}
\end{aligned}
$$

Thus $\mathfrak{F}: \mathcal{U}\left(C_{2}^{+}\right) \rightarrow \mathfrak{Z}_{\mathfrak{q}}, e_{1} \mapsto \xi_{1}, e_{2} \mapsto \xi_{2}$, is an isomorphism of algebras. (Of course $C_{2} \simeq B_{2}$ but in higher rank we will get different root systems depending on the parity of $N$ ).

Row 4. Let $q \in \mathbb{G}_{N}^{\prime}, N \neq 2,4$. These diagrams correspond to braidings of super type $B$ with $\Delta_{\mathfrak{q}}^{+}=\left\{\alpha_{1}, 2 \alpha_{1}+\alpha_{2}, \alpha_{1}+\alpha_{2}, \alpha_{2}\right\}$.

If the diagram is $\stackrel{q}{q} q^{-2}-1$, then the Cartan roots are $\alpha_{1}$ and $\alpha_{1}+\alpha_{2}$, with $N_{\alpha_{1}}=N, N_{\alpha_{1}+\alpha_{2}}=M$; here, $M=N$ if $N$ is odd and $M=\frac{N}{2}$ if $N$ is even. The elements $x_{1}^{N}, x_{12}^{M} \in \widetilde{\mathcal{B}}_{\mathfrak{q}}$ are primitive in $\widetilde{\mathcal{B}}_{\mathfrak{q}}$. Thus, in $\mathfrak{Z}_{\mathfrak{q}}$, $\left[\xi_{12}, \xi_{1}\right]=0$ and $\mathfrak{Z}_{\mathfrak{q}} \simeq \mathcal{U}\left(\left(A_{1} \oplus A_{1}\right)^{+}\right)$.

If we consider the diagram $\stackrel{-q^{-1} q^{2}-1}{\circ}$, then $\mathfrak{O}_{\mathfrak{q}}=\left\{\alpha_{1}, \alpha_{1}+\alpha_{2}\right\}, N_{\alpha_{1}}=M$ and $N_{\alpha_{1}+\alpha_{2}}=N$. The elements $x_{1}^{M}, x_{12}^{N} \in \widetilde{\mathcal{B}}_{\mathfrak{q}}$ are primitive, so $\left[\xi_{12}, \xi_{1}\right]=0$ and $\mathfrak{Z}_{\mathfrak{q}} \simeq \mathcal{U}\left(\left(A_{1} \oplus A_{1}\right)^{+}\right)$.

Row 5. Let $q \in \mathbb{G}_{N}^{\prime}, N \neq 3, \zeta \in \mathbb{G}_{3}^{\prime}$. The diagram ${ }_{\bigcirc}^{\zeta} q^{-1} q$ corresponds to a braiding of standard type $B_{2}$, so $\Delta_{\mathfrak{q}}^{+}=\left\{\alpha_{1}, 2 \alpha_{1}+\alpha_{2}, \alpha_{1}+\alpha_{2}, \alpha_{2}\right\}$. The other diagram $\underset{\sim}{\zeta} q \zeta^{-1} \zeta q^{-1}$ is obtained by changing the parameter $q \leftrightarrow \zeta q^{-1}$.

The Cartan roots are $2 \alpha_{1}+\alpha_{2}$ and $\alpha_{2}$, with $N_{2 \alpha_{1}+\alpha_{2}}=M:=\operatorname{ord}\left(\zeta q^{-1}\right)$ and $N_{\alpha_{2}}=N$. The elements $x_{112}^{M}, x_{2}^{N} \in \widetilde{\mathcal{B}}_{\mathfrak{q}}$ are primitive. Thus, in $\mathfrak{Z}_{\mathfrak{q}}$, we have $\left[\xi_{112}, \xi_{2}\right]=0$. Hence, $\mathfrak{Z}_{\mathfrak{q}} \simeq \mathcal{U}\left(\left(A_{1} \oplus A_{1}\right)^{+}\right)$.

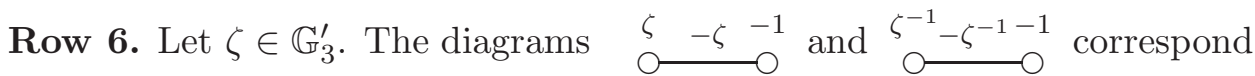
to braidings of standard type $B$, thus $\Delta_{\mathfrak{q}}^{+}=\left\{\alpha_{1}, 2 \alpha_{1}+\alpha_{2}, \alpha_{1}+\alpha_{2}, \alpha_{2}\right\}$. In both cases $\mathfrak{O}_{\mathfrak{q}}$ is empty so the corresponding Lie algebras are trivial.

Row 7. Let $\zeta \in \mathbb{G}_{12}^{\prime}$. The diagrams of this row correspond to braidings of type $\mathfrak{u} \mathfrak{f o}(7)$. In all cases $\mathfrak{O}_{\mathfrak{q}}=\emptyset$ and the associated Lie algebras are trivial.

Row 8. Let $\zeta \in \mathbb{G}_{12}^{\prime}$. The diagrams of this row correspond to braidings of type $\mathfrak{u f o}(8)$. For $\underset{-\zeta^{2}}{\mathrm{O}} \boldsymbol{\zeta}_{-}^{-\zeta^{2}}, \Delta_{\mathfrak{q}}^{+}=\left\{\alpha_{1}, 2 \alpha_{1}+\alpha_{2}, \alpha_{1}+\alpha_{2}, \alpha_{1}+2 \alpha_{2}, \alpha_{2}\right\}$. In this case $\mathfrak{O}_{\mathfrak{q}}=\left\{\alpha_{1}+\alpha_{2}\right\}, N_{\alpha_{1}+\alpha_{2}}=12$. Hence $\mathfrak{Z}_{\mathfrak{q}} \simeq \mathcal{U}\left(A_{1}^{+}\right)$. The same result holds for the other braidings in this row.

Row 9. Let $\zeta \in \mathbb{G}_{9}^{\prime}$. The diagrams of this row correspond to braidings of type $\mathfrak{b} \mathfrak{r j}(2 ; 3)$. If $\mathfrak{q}$ has diagram \begin{tabular}{cccc}
$-\zeta$ & $\zeta^{7}$ & $\zeta^{3}$ \\
\hdashline & - & &
\end{tabular} , then

$$
\Delta_{\mathfrak{q}}^{+}=\left\{\alpha_{1}, 2 \alpha_{1}+\alpha_{2}, 3 \alpha_{1}+2 \alpha_{2}, \alpha_{1}+\alpha_{2}, \alpha_{1}+2 \alpha_{2}, \alpha_{2}\right\} .
$$


In this case $\mathfrak{O}_{\mathfrak{q}}=\left\{\alpha_{1}, \alpha_{1}+\alpha_{2}\right\}$ and $N_{\alpha_{1}}=N_{\alpha_{1}+\alpha_{2}}=18$. Thus $\left[\xi_{12}, \xi_{1}\right]=0$, so $\mathfrak{Z}_{\mathfrak{q}} \simeq \mathcal{U}\left(\left(A_{1} \oplus A_{1}\right)^{+}\right)$.

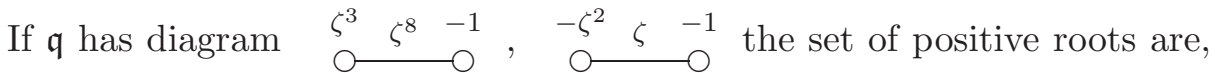
respectively,

$$
\begin{aligned}
& \left\{\alpha_{1}, 2 \alpha_{1}+\alpha_{2}, 3 \alpha_{1}+2 \alpha_{2}, 4 \alpha_{1}+3 \alpha_{2}, \alpha_{1}+\alpha_{2}, \alpha_{2}\right\}, \\
& \left\{\alpha_{1}, 4 \alpha_{1}+\alpha_{2}, 3 \alpha_{1}+\alpha_{2}, 2 \alpha_{1}+\alpha_{2}, \alpha_{1}+\alpha_{2}, \alpha_{2}\right\}
\end{aligned}
$$

the Cartan roots are, respectively, $\alpha_{1}+\alpha_{2}, 2 \alpha_{1}+\alpha_{2}$ and $\alpha_{1}, 2 \alpha_{1}+\alpha_{2}$. Hence, in both cases, $\mathfrak{Z}_{\mathfrak{q}} \simeq \mathcal{U}\left(\left(A_{1} \oplus A_{1}\right)^{+}\right)$.

Row 10. Let $q \in \mathbb{G}_{N}^{\prime}, N \geq 4$. The diagram $\underset{\sim}{q} q^{-3} q^{3}$ corresponds to a braiding of Cartan type $G_{2}$, so $\mathfrak{O}_{\mathfrak{q}}=\Delta_{\mathfrak{q}}^{+}=\left\{\alpha_{1}, \alpha_{1}+\alpha_{2}, 2 \alpha_{1}+\alpha_{2}, 3 \alpha_{1}+\right.$ $\left.\alpha_{2}, 3 \alpha_{1}+2 \alpha_{2}, \alpha_{2}\right\}$. The coproducts of the PBW-generators are:

$$
\begin{aligned}
& \underline{\Delta}\left(x_{1}\right)=x_{1} \otimes 1+1 \otimes x_{1} ; \quad \underline{\Delta}\left(x_{2}\right)=x_{2} \otimes 1+1 \otimes x_{2} ; \\
& \underline{\Delta}\left(x_{12}\right)=x_{12} \otimes 1+1 \otimes x_{12}+\left(1-q^{-3}\right) x_{1} \otimes x_{2} ; \\
& \underline{\Delta}\left(x_{112}\right)=x_{112} \otimes 1+1 \otimes x_{112}+(1+q)\left(1-q^{-2}\right) x_{1} \otimes x_{12} \\
& \quad+\left(1-q^{-2}\right)\left(1-q^{-3}\right) x_{1}^{2} \otimes x_{2} ; \\
& \Delta\left(x_{1112}\right)=x_{1112} \otimes 1+1 \otimes x_{1112}+q^{2}\left(1-q^{-3}\right) x_{1} \otimes x_{112} \\
& \quad+\left(q^{2}-1\right)\left(1-q^{-3}\right) x_{1}^{2} \otimes x_{12}+\left(1-q^{-3}\right)\left(1-q^{-2}\right)\left(1-q^{-1}\right) x_{1}^{3} \otimes x_{2} ; \\
& \underline{\Delta}\left(\left[x_{112}, x_{12}\right]_{c}\right)=\left[x_{112}, x_{12}\right]_{c} \otimes 1+1 \otimes\left[x_{112}, x_{12}\right]_{c}+\left(q-q^{-1}\right) x_{112} \otimes x_{12} \\
& \quad+\left(1-q^{-3}\right)(1+q)\left(1-q^{-1}+q\right) x_{112} x_{1} \otimes x_{2} \\
& \quad-q q_{21}\left(1-q^{-3}\right)\left(1+q-q^{2}\right) x_{1112} \otimes x_{2}+q^{2} q_{21}\left(1-q^{-3}\right) x_{1} \otimes\left[x_{112}, x_{2}\right]_{c} \\
& \quad+\left(1-q^{-3}\right)^{2}\left(q^{2}-1\right) x_{1}^{2} \otimes x_{2} x_{12} \\
& \quad+q_{21}\left(1-q^{-3}\right)^{2}\left(1-q^{-2}\right)\left(1-q^{-1}\right) x_{1}^{3} \otimes x_{2}^{2} .
\end{aligned}
$$

We have two cases.

(1) If 3 does not divide $N$, then $N_{\beta}=N$ for all $\beta \in \Delta_{\mathfrak{q}}^{+}$. Thus, in $\widetilde{\mathcal{B}}_{\mathfrak{q}}$,

$$
\begin{aligned}
& \Delta\left(x_{1}^{N}\right)=x_{1}^{N} \otimes 1+1 \otimes x_{1}^{N} ; \quad \underline{\Delta}\left(x_{2}^{N}\right)=x_{2}^{N} \otimes 1+1 \otimes x_{2}^{N} ; \\
& \Delta\left(x_{12}^{N}\right)=x_{12}^{N} \otimes 1+1 \otimes x_{12}^{N}+a_{1} x_{1}^{N} \otimes x_{2}^{N} ; \\
& \triangleq \\
& \left(x_{112}^{N}\right)=x_{112}^{N} \otimes 1+1 \otimes x_{112}^{N}+a_{2} x_{1}^{N} \otimes x_{12}^{N}+a_{3} x_{1}^{2 N} \otimes x_{2}^{N} ; \\
& \underline{\Delta}\left(x_{1112}^{N}\right)=x_{1112}^{N} \otimes 1+1 \otimes x_{1112}^{N}+a_{4} x_{1}^{N} \otimes x_{112}^{N}+a_{5} x_{1}^{2 N} \otimes x_{12}^{N} \\
& \quad+a_{6} x_{1}^{3 N} \otimes x_{2}^{N} ; \\
& \underline{\Delta}\left(\left[x_{112}, x_{12}\right]_{c}^{N}\right)=\left[x_{112}, x_{12}\right]_{c}^{N} \otimes 1+1 \otimes\left[x_{112}, x_{12}\right]_{c}^{N}+a_{7} x_{112}^{N} \otimes x_{12}^{N} \\
& \quad+a_{8} x_{1112}^{N} \otimes x_{2}^{N}+a_{9} x_{1}^{N} \otimes x_{12}^{2 N}+a_{10} x_{1}^{2 N} \otimes x_{2}^{N} x_{12}^{N} \\
& \quad+a_{11} x_{112}^{N} x_{1}^{N} \otimes x_{2}^{N}+a_{12} x_{1}^{3 N} \otimes x_{2}^{2 N} ;
\end{aligned}
$$


for some $a_{i} \in \mathbf{k}$. Since

$$
\begin{aligned}
a_{1} & =\left(1-q^{-3}\right)^{N} q_{21}^{\frac{N(N-1)}{2}} \neq 0, \\
a_{3} & =\left(1-q^{-2}\right)^{N}\left(1-q^{-3}\right)^{N} \neq 0, \\
a_{6} & =\left(1-q^{-1}\right)^{N}\left(1-q^{-2}\right)^{N}\left(1-q^{-3}\right)^{N} q_{21}^{\frac{3 N(N-1)}{2}} \neq 0, \\
a_{12} & =\left(1-q^{-1}\right)^{N}\left(1-q^{-2}\right)^{N}\left(1-q^{-3}\right)^{2 N} \neq 0,
\end{aligned}
$$

the elements $x_{12}^{N}, x_{112}^{N}, x_{1112}^{N}$ and $\left[x_{112}, x_{12}\right]_{c}^{N}$ are not primitive. Hence $\mathfrak{Z}_{\mathfrak{q}}$ is generated by $\xi_{1}$ and $\xi_{2}$; also

$$
\begin{aligned}
{\left[\xi_{2}, \xi_{1}\right] } & =a_{1} \xi_{12} ; & {\left[\xi_{12}, \xi_{1}\right] } & =a_{2} \xi_{112} ; \\
{\left[\xi_{112}, \xi_{1}\right] } & =a_{4} \xi_{1112} ; & {\left[\xi_{1}, \xi_{1112}\right] } & =\left[\xi_{2}, \xi_{12}\right]=0 .
\end{aligned}
$$

Thus, we have $\mathfrak{Z}_{\mathfrak{q}} \simeq \mathcal{U}\left(G_{2}^{+}\right)$.

(2) If $N=3 M$, then $N_{\alpha_{1}}=N_{\alpha_{1}+\alpha_{2}}=N_{2 \alpha_{1}+\alpha_{2}}=N$ and $N_{3 \alpha_{1}+\alpha_{2}}=$ $N_{3 \alpha_{1}+2 \alpha_{2}}=N_{\alpha_{2}}=M$. In this case we have

$$
\begin{aligned}
& \underline{\Delta}\left(x_{1}^{N}\right)=x_{1}^{N} \otimes 1+1 \otimes x_{1}^{N} ; \quad \underline{\Delta}\left(x_{2}^{M}\right)=x_{2}^{M} \otimes 1+1 \otimes x_{2}^{M} ; \\
& \Delta\left(x_{12}^{N}\right)=x_{12}^{N} \otimes 1+1 \otimes x_{12}^{N}+\left(1-q^{-3}\right)^{M} q_{21}^{\frac{N(M-1)}{2}}\left[x_{112}, x_{12}\right]_{c}^{M} \otimes x_{2}^{M} \\
& +\left(1-q^{-3}\right)^{2 M} x_{1112}^{M} \otimes x_{2}^{2 M}+\left(1-q^{-3}\right)^{N} q_{21}^{\frac{N(N-1)}{2}} x_{1}^{N} \otimes x_{2}^{3 M} ; \\
& \underline{\Delta}\left(x_{112}^{N}\right)=x_{112}^{M} \otimes 1+1 \otimes x_{112}^{M}+b_{1} x_{1}^{N} \otimes x_{12}^{N}+b_{2} x_{1112}^{M} \otimes\left[x_{112}, x_{12}\right]_{c}^{M} \\
& +b_{3} x_{1}^{2 N} \otimes x_{2}^{3 M}+b_{4} x_{1112}^{2 M} \otimes x_{2}^{M} \\
& +b_{5} x_{1112}^{M} x_{1}^{N} \otimes x_{2}^{2 M}+b_{6} x_{1}^{N} \otimes x_{2}^{M} ; \\
& \Delta\left(x_{112}^{M}\right)=x_{112}^{M} \otimes 1+1 \otimes x_{112}^{M}+b_{7} x_{1}^{N} \otimes x_{2}^{M}\left[x_{112}, x_{12}\right]_{c}^{M} ; \\
& \underline{\Delta}\left(\left[x_{112}, x_{12}\right]_{c}^{M}\right)=x_{112}^{M} \otimes 1+1 \otimes x_{112}^{M}+b_{8} x_{1}^{N} \otimes x_{2}^{2 M}+b_{9} x_{1112}^{M} \otimes x_{2}^{M} \text {; }
\end{aligned}
$$

for some $b_{i} \in \mathbf{k}$. We compute some of them explicitly:

$$
\begin{aligned}
& b_{2}=(1+q)^{M}\left(1-q^{-2}\right)^{M} q^{2 M} q_{21}^{\frac{N(M-1)}{2}}, \\
& b_{7}=\left(1-q^{-3}\right)^{M}\left(1-q^{-2}\right)^{M}\left(1-q^{-1}\right)^{M} q_{21}^{\frac{N(M-1)}{2}}, \\
& b_{8}=\left(1-q^{-3}\right)^{2 M}\left(1-q^{-2}\right)^{M}\left(1-q^{-1}\right)^{M} q_{21}^{M} .
\end{aligned}
$$

As these scalars are not zero, the elements $x_{12}^{N}, x_{112}^{N}, x_{1112}^{M}$ and $\left[x_{112}, x_{12}\right]_{c}^{M}$ are not primitive. Thus $\mathfrak{Z}_{\mathfrak{q}} \simeq \mathcal{U}\left(G_{2}^{+}\right)$.

Row 11. Let $\zeta \in \mathbb{G}_{8}^{\prime}$. The diagrams of this row correspond to braidings of standard type $G_{2}$, so $\Delta_{\mathfrak{q}}^{+}=\left\{\alpha_{1}, 3 \alpha_{1}+\alpha_{2}, 2 \alpha_{1}+\alpha_{2}, 3 \alpha_{1}+2 \alpha_{2}, \alpha_{1}+\alpha_{2}, \alpha_{2}\right\}$.

If $\mathfrak{q}$ has diagram $\underset{\zeta^{2}}{\stackrel{\zeta}{\circ}} \stackrel{\zeta^{-1}}{\circ}$, then the Cartan roots are $2 \alpha_{1}+\alpha_{2}$ and $\alpha_{2}$ with $N_{2 \alpha_{1}+\alpha_{2}}=N_{\alpha_{2}}=8$. The elements $x_{112}^{8}, x_{2}^{8} \in \widetilde{\mathcal{B}}_{\mathfrak{q}}$ are primitive and $\left[\xi_{2}, \xi_{112}\right]=0$ in $\mathfrak{Z}_{\mathfrak{q}}$. Hence $\mathfrak{Z}_{\mathfrak{q}} \simeq \mathcal{U}\left(\left(A_{1} \oplus A_{1}\right)^{+}\right)$. An analogous result holds for the other diagrams of the row. 
Row 12. Let $\zeta \in \mathbb{G}_{24}^{\prime}$. This row corresponds to type $\mathfrak{u} \mathfrak{f o}(9)$. If $\mathfrak{q}$ has

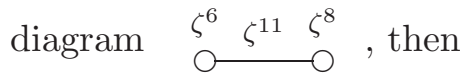

$\Delta_{\mathfrak{q}}^{+}=\left\{\alpha_{1}, 3 \alpha_{1}+\alpha_{2}, 2 \alpha_{1}+\alpha_{2}, 3 \alpha_{1}+2 \alpha_{2}, 4 \alpha_{1}+3 \alpha_{2}, \alpha_{1}+\alpha_{2}, \alpha_{1}+2 \alpha_{2}, \alpha_{2}\right\}$ and $\mathfrak{O}_{\mathfrak{q}}=\left\{\alpha_{1}+\alpha_{2}, 3 \alpha_{1}+\alpha_{2}\right\}$. Here, $N_{\alpha_{1}+\alpha_{2}}=N_{3 \alpha_{1}+\alpha_{2}}=24$, and $x_{12}^{24}, x_{1112}^{24} \in \widetilde{\mathcal{B}}_{\mathfrak{q}}$ are primitive. In $\mathfrak{Z}_{\mathfrak{q}}$ we have the relation $\left[\xi_{12}, \xi_{1112}\right]=0$; thus $\mathfrak{Z}_{\mathfrak{q}} \simeq \mathcal{U}\left(\left(A_{1} \oplus A_{1}\right)^{+}\right)$.

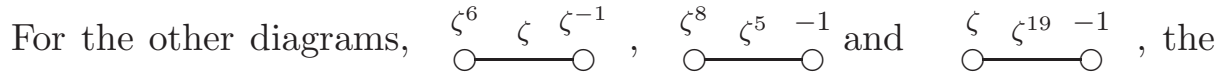
sets of positive roots are, respectively,

$$
\begin{aligned}
& \left\{\alpha_{1}, \alpha_{1}+\alpha_{2}, 2 \alpha_{1}+\alpha_{2}, 3 \alpha_{1}+\alpha_{2}, 3 \alpha_{1}+2 \alpha_{2}, 5 \alpha_{1}+2 \alpha_{2}, 5 \alpha_{1}+3 \alpha_{2}, \alpha_{2}\right\}, \\
& \left\{\alpha_{1}, \alpha_{1}+\alpha_{2}, 2 \alpha_{1}+\alpha_{2}, 3 \alpha_{1}+2 \alpha_{2}, 4 \alpha_{1}+3 \alpha_{2}, 5 \alpha_{1}+3 \alpha_{2}, 5 \alpha_{1}+4 \alpha_{2}, \alpha_{2}\right\}, \\
& \left\{\alpha_{1}, \alpha_{1}+\alpha_{2}, 2 \alpha_{1}+\alpha_{2}, 3 \alpha_{1}+\alpha_{2}, 4 \alpha_{1}+\alpha_{2}, 5 \alpha_{1}+\alpha_{2}, 5 \alpha_{1}+2 \alpha_{2}, \alpha_{2}\right\} .
\end{aligned}
$$

The Cartan roots are, respectively, $2 \alpha_{1}+\alpha_{2}, \alpha_{2} ; \alpha_{1}+\alpha_{2}, 5 \alpha_{1}+3 \alpha_{2} ; \alpha_{1}, 5 \alpha_{1}+$ $2 \alpha_{2}$. Hence, in all cases, $\mathfrak{Z}_{\mathfrak{q}} \simeq \mathcal{U}\left(\left(A_{1} \oplus A_{1}\right)^{+}\right)$.

Row 13. Let $\zeta \in \mathbb{G}_{5}^{\prime}$. The braidings in this row are associated to the

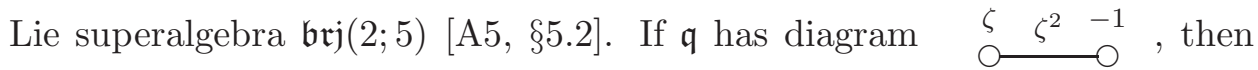
$\Delta_{\mathfrak{q}}^{+}=\left\{\alpha_{1}, 3 \alpha_{1}+\alpha_{2}, 2 \alpha_{1}+\alpha_{2}, 5 \alpha_{1}+3 \alpha_{2}, 3 \alpha_{1}+2 \alpha_{2}, 4 \alpha_{1}+3 \alpha_{2}, \alpha_{1}+\alpha_{2}, \alpha_{2}\right\}$. In this case the Cartan roots are $\alpha_{1}, \alpha_{1}+\alpha_{2}, 2 \alpha_{1}+\alpha_{2}$ and $3 \alpha_{1}+\alpha_{2}$, with $N_{\alpha_{1}}=N_{3 \alpha_{1}+2 \alpha_{2}}=5$ and $N_{\alpha_{1}+\alpha_{2}}=N_{2 \alpha_{1}+\alpha_{2}}=10$. In $\widetilde{\mathcal{B}}_{\mathfrak{q}}$,

$$
\begin{aligned}
& \Delta\left(x_{1}\right)=x_{1} \otimes 1+1 \otimes x_{1} ; \\
& \Delta\left(x_{12}\right)=x_{12} \otimes 1+1 \otimes x_{12}+\left(1-\zeta^{2}\right) x_{1} \otimes x_{2} ; \\
& \Delta\left(x_{112}\right)=x_{112} \otimes 1+1 \otimes x_{112}+(1+\zeta)\left(1-\zeta^{3}\right) x_{1} \otimes x_{12} \\
& \quad+\left(1-\zeta^{2}\right)\left(1-\zeta^{3}\right) x_{1}^{2} \otimes x_{2} ; \\
& \Delta\left(\left[x_{112}, x_{12}\right]_{c}\right)=\left[x_{112}, x_{12}\right]_{c} \otimes 1+1 \otimes\left[x_{112}, x_{12}\right]_{c} \\
& \quad-\zeta^{3}\left(1-\zeta^{3}\right)(1+\zeta)^{2} x_{1} \otimes x_{12}^{2}-\zeta q_{21} x_{1} x_{112} \otimes x_{2} \\
& \quad+\left(1+q_{21}+\zeta^{3} q_{21}\right) x_{112} x_{1} \otimes x_{2}+\zeta\left(1-\zeta^{2}\right) x_{1} x_{12} x_{1} \otimes x_{2} \\
& \quad+\left(1-\zeta^{2}\right)\left(1-\zeta^{3}\right)^{2} x_{1}^{2} \otimes x_{2} x_{12} .
\end{aligned}
$$

Hence the coproducts of $x_{1}^{5}, x_{12}^{10}, x_{112}^{10},\left[x_{112}, x_{12}\right]_{c}^{5}, \in \widetilde{\mathcal{B}}_{\mathfrak{q}}$ are:

$$
\begin{aligned}
& \underline{\Delta}\left(x_{1}^{5}\right)=x_{1}^{5} \otimes 1+1 \otimes x_{1}^{5} ; \quad \underline{\Delta}\left(x_{12}^{10}\right)=x_{12}^{10} \otimes 1+1 \otimes x_{12}^{10} ; \\
& \underline{\Delta}\left(x_{112}^{10}\right)=x_{112}^{10} \otimes 1+1 \otimes x_{112}^{10}+a_{1} x_{1}^{10} \otimes x_{12}^{10}+a_{2} x_{1}^{5} \otimes\left[x_{112}, x_{12}\right]_{c}^{5} ; \\
& \underline{\Delta}\left(\left[x_{112}, x_{12}\right]_{c}^{5}\right)=\left[x_{112}, x_{12}\right]_{c}^{5} \otimes 1+1 \otimes\left[x_{112}, x_{12}\right]_{c}^{5}+a_{3} x_{1}^{5} \otimes x_{12}^{10} .
\end{aligned}
$$

for some $a_{i} \in \mathbf{k}$. Thus, the following relations hold in $\mathfrak{Z}_{\mathfrak{q}}$

$$
\left[\xi_{12}, \xi_{1}\right]=a_{3} \xi_{112,12} ; \quad\left[\xi_{112,12}, \xi_{1}\right]=a_{2} \xi_{112} ; \quad\left[\xi_{1}, \xi_{112,12}\right]=\left[\xi_{12}, \xi_{112}\right]=0 .
$$


Since

$$
\begin{aligned}
& a_{1}=-\left(1-\zeta^{3}\right)^{5}(1+\zeta)^{5}\left(1+62 \zeta-15 \zeta^{2}-87 \zeta^{3}+70 \zeta^{4}\right) \neq 0 \\
& a_{3}=-\left(1-\zeta^{3}\right)^{5}(1+\zeta)^{8}\left(4-8 \zeta-19 \zeta^{2}-3 \zeta^{3}-50 \zeta^{4}\right) \neq 0,
\end{aligned}
$$

the elements $x_{112}^{10},\left[x_{112}, x_{12}\right]_{c}^{5}$ are not primitive, so $\xi_{1}, \xi_{12}$ generate $\mathfrak{Z}_{\mathfrak{q}}$. Hence, $\mathfrak{Z}_{\mathfrak{q}} \simeq \mathcal{U}\left(B_{2}^{+}\right)$.

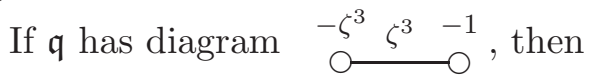

$\Delta_{\mathfrak{q}}^{+}=\left\{\alpha_{1}, 4 \alpha_{1}+\alpha_{2}, 3 \alpha_{1}+\alpha_{2}, 5 \alpha_{1}+2 \alpha_{2}, 2 \alpha_{1}+\alpha_{2}, 3 \alpha_{1}+2 \alpha_{2}, \alpha_{1}+\alpha_{2}, \alpha_{2}\right\}$, $\mathfrak{O}_{\mathfrak{q}}=\left\{\alpha_{1}, 3 \alpha_{1}+\alpha_{2}, 2 \alpha_{1}+\alpha_{2}, \alpha_{1}+\alpha_{2}\right\}$,

with $N_{\alpha_{1}}=N_{\alpha_{1}+\alpha_{2}}=10, N_{3 \alpha_{1}+\alpha_{2}}=N_{\alpha_{1}+\alpha_{2}}=5$. The generators of $\mathfrak{Z}_{\mathfrak{q}}$ are $\xi_{1}$ and $\xi_{12}$ and they satisfy the following relations

$$
\left[\xi_{12}, \xi_{1}\right]=b_{1} \xi_{1112}, \quad\left[\xi_{1112}, \xi_{12}\right]=b_{2} \xi_{112}, \quad\left[\xi_{1}, \xi_{1112}\right]=\left[\xi_{12}, \xi_{112}\right]=0,
$$

for some $b_{1}, b_{2} \in \mathbf{k}^{\times}$. Hence $\mathfrak{Z}_{\mathfrak{q}} \simeq \mathcal{U}\left(C_{2}^{+}\right)$.

Row 14. Let $\zeta \in \mathbb{G}_{20}^{\prime}$. This row corresponds to type $\mathfrak{u} \mathfrak{f} \mathfrak{o}(10)$. If $\mathfrak{q}$ has

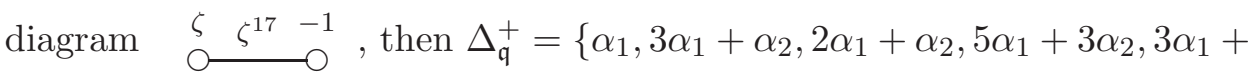
$\left.2 \alpha_{2}, 4 \alpha_{1}+3 \alpha_{2}, \alpha_{1}+\alpha_{2}, \alpha_{2}\right\}$. The Cartan roots are $\alpha_{1}$ and $3 \alpha_{1}+2 \alpha_{2}$ with $N_{\alpha_{1}}=N_{3 \alpha_{1}+2 \alpha_{2}}=20$. The elements $x_{1}^{20},\left[x_{112}, x_{12}\right]_{c}^{20} \in \widetilde{\mathcal{B}}_{\mathfrak{q}}$ are primitive; thus $\left[\xi_{12}, \xi_{112,12}\right]=0$ in $\mathfrak{Z}_{\mathfrak{q}}$ and $\mathfrak{Z}_{\mathfrak{q}} \simeq \mathcal{U}\left(\left(A_{1} \oplus A_{1}\right)^{+}\right)$. The same holds when the diagram of $\mathfrak{q}$ is another one in this row: $\mathfrak{Z}_{\mathfrak{q}} \simeq \mathcal{U}\left(\left(A_{1} \oplus A_{1}\right)^{+}\right)$.

Row 15. Let $\zeta \in \mathbb{G}_{15}^{\prime}$. This row corresponds to type $\mathfrak{u} \mathfrak{f} \mathfrak{o}(11)$. If $\mathfrak{q}$ has diagram $\underset{\sim}{\stackrel{-\zeta}{-}-\zeta^{12} \zeta^{5}}$, then $\Delta_{\mathfrak{q}}^{+}=\left\{\alpha_{1}, 3 \alpha_{1}+\alpha_{2}, 5 \alpha_{1}+2 \alpha_{2}, 2 \alpha_{1}+\alpha_{2}, 3 \alpha_{1}+\right.$ $\left.2 \alpha_{2}, \alpha_{1}+\alpha_{2}, \alpha_{1}+2 \alpha_{2}, \alpha_{2}\right\}$. The Cartan roots are $\alpha_{1}$ and $3 \alpha_{1}+2 \alpha_{2}$ with $N_{\alpha_{1}}=N_{3 \alpha_{1}+2 \alpha_{2}}=30$. In $\mathfrak{Z}_{\mathfrak{q}}$ we have $\left[\xi_{12}, \xi_{112,12}\right]=0$, thus $\mathfrak{Z}_{\mathfrak{q}} \simeq \mathcal{U}\left(\left(A_{1} \oplus\right.\right.$ $\left.\left.A_{1}\right)^{+}\right)$. The same result holds if we consider the other diagrams of this row.

Row 16. Let $\zeta \in \mathbb{G}_{7}^{\prime}$. This row corresponds to type $\mathfrak{u f o}(12)$. If $\mathfrak{q}$ has

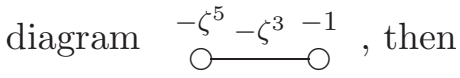

$$
\begin{aligned}
\Delta_{\mathfrak{q}}^{+}=\left\{\alpha_{1}, 5 \alpha_{1}+\alpha_{2}, 4 \alpha_{1}+\alpha_{2}, 7 \alpha_{1}+2 \alpha_{2}, 3 \alpha_{1}+\alpha_{2}, 8 \alpha_{1}+3 \alpha_{2},\right. \\
\left.5 \alpha_{1}+2 \alpha_{2}, 7 \alpha_{1}+3 \alpha_{2}, 2 \alpha_{1}+\alpha_{2}, 3 \alpha_{1}+2 \alpha_{2}, \alpha_{1}+\alpha_{2}, \alpha_{2}\right\} .
\end{aligned}
$$

Also, $\mathfrak{O}_{\mathfrak{q}}=\left\{\alpha_{1}, 4 \alpha_{1}+\alpha_{2}, 3 \alpha_{1}+\alpha_{2}, 5 \alpha_{1}+2 \alpha_{2}, 2 \alpha_{1}+\alpha_{2}, \alpha_{1}+\alpha_{2}\right\}$ with $N_{\beta}=14$ for all $\beta \in \mathfrak{O}_{\mathfrak{q}}$. In $\widetilde{\mathcal{B}}_{\mathfrak{q}}$ we have

$$
\begin{aligned}
& \underline{\Delta}\left(x_{1}\right)=x_{1} \otimes 1+1 \otimes x_{1} ; \\
& \underline{\Delta}\left(x_{12}\right)=x_{12} \otimes 1+1 \otimes x_{12}+\left(1+\zeta^{3}\right) x_{1} \otimes x_{2} ; \\
& \underline{\Delta}\left(x_{112}\right)=x_{112} \otimes 1+1 \otimes x_{112}+(1-\zeta)\left(1-\zeta^{5}\right) x_{1} \otimes x_{12} \\
& \quad+(1-\zeta)\left(1+\zeta^{3}\right) x_{1}^{2} \otimes x_{2} ;
\end{aligned}
$$




$$
\begin{aligned}
& \underline{\Delta}\left(x_{1112}\right)=x_{1112} \otimes 1+1 \otimes x_{1112}+\left(1+\zeta^{3}-\zeta^{5}\right)\left(1+\zeta^{6}\right) x_{1} \otimes x_{112} \\
& +\zeta\left(\zeta^{3}-1\right) x_{1}^{2} \otimes x_{12}+\zeta^{6}\left(1-\zeta^{2}\right)\left(1+\zeta^{3}\right) x_{1}^{3} \otimes x_{2} ; \\
& \underline{\Delta}\left(x_{11112}\right)=x_{11112} \otimes 1+1 \otimes x_{11112}-\zeta(1-\zeta)\left(1-\zeta^{2}\right) x_{1} \otimes x_{1112} \\
& +\left(1-\zeta^{4}\right) x_{1}^{2} \otimes x_{112}-(1-\zeta)\left(1-\zeta^{2}\right)^{2} x_{1}^{3} \otimes x_{12} \\
& +\zeta^{2}(1-\zeta)\left(1-\zeta^{2}\right) x_{1}^{4} \otimes x_{2} \\
& \underline{\Delta}\left(\left[x_{1112}, x_{112}\right]_{c}\right)=\left[x_{1112}, x_{112}\right]_{c} \otimes 1+1 \otimes\left[x_{1112}, x_{112}\right]_{c} \\
& -\frac{\left(1-\zeta^{5}\right)}{(1+\zeta)}\left(1-\zeta^{3}+2 \zeta^{4}\right) x_{1} \otimes x_{112}^{2} \\
& -q_{21}(1-\zeta)\left(1-\zeta^{3}\right) x_{1}^{2} \otimes\left[x_{112}, x_{12}\right]_{c} \\
& -(1-\zeta)^{2}\left(4+4 \zeta+\zeta^{2}-2 \zeta^{3}-3 \zeta^{4}\right) x_{1}^{2} \otimes x_{12} x_{112} \\
& +q_{21}\left(1-\zeta^{2}\right)^{2} \zeta^{4}\left(1-2 \zeta-3 \zeta^{4}-2 \zeta^{5}+\zeta^{6}\right) x_{1}^{3} \otimes x_{12}^{2} \\
& +(1-\zeta)^{2}\left(1+\zeta^{3}\right)^{2}\left(1+\zeta^{6}\right) x_{1}^{3} \otimes x_{2} x_{112}-\zeta(1-\zeta)\left(1-\zeta^{2}\right) x_{1112} \otimes x_{112} \\
& -q_{21} \zeta^{6}(1-\zeta)^{2}\left(1-\zeta^{2}\right)(1+2 \zeta) x_{1}^{4} \otimes x_{2} x_{12} \\
& +q_{21}^{2} \zeta^{2}(1-\zeta)^{2}\left(1-\zeta^{2}\right)\left(1+\zeta^{3}\right) x_{1}^{5} \otimes x_{2}^{2} \\
& -q_{12}^{2}\left(1+\zeta^{3}\right)(1-\zeta)\left(1-\zeta^{4}+\zeta^{6}\right) x_{111112} \otimes x_{2} \\
& +\zeta q_{21}\left(1+\zeta^{3}\right)(1-\zeta)\left(1-\zeta^{2}\right)\left(1+\zeta-\zeta^{2}\right) x_{11112} x_{1} \otimes x_{2} \\
& -\zeta(1-\zeta)^{2}\left(1+\zeta^{3}\right)\left(1-\zeta-2 \zeta^{2}-\zeta^{3}\right) x_{1112} x_{1}^{2} \otimes x_{2} \\
& +(1-\zeta)\left(1+\zeta^{2}+\zeta^{3}-\zeta^{4}-\zeta^{5}\right) x_{1112} x_{1} \otimes x_{12} \\
& +\zeta q_{21}(1-\zeta)^{2}\left(2+\zeta-\zeta^{3}\right) x_{11112} \otimes x_{12} .
\end{aligned}
$$

Hence

$$
\begin{aligned}
& \underline{\Delta}\left(x_{1}^{14}\right)=x_{1}^{14} \otimes 1+1 \otimes x_{1}^{14} ; \quad \underline{\Delta}\left(x_{12}^{14}\right)=x_{12}^{14} \otimes 1+1 \otimes x_{12}^{14} ; \\
& \underline{\Delta}\left(x_{112}^{14}\right)=x_{112}^{14} \otimes 1+1 \otimes x_{112}^{14}+a_{1} x_{1}^{14} \otimes x_{12}^{14} \text {; } \\
& \underline{\Delta}\left(x_{1112}^{14}\right)=x_{1112}^{14} \otimes 1+1 \otimes x_{1112}^{14}+a_{2} x_{1}^{14} \otimes x_{112}^{14}+a_{3} x_{1}^{28} \otimes x_{12}^{14} ; \\
& \Delta\left(x_{1112}^{14}\right)=x_{11112}^{14} \otimes 1+1 \otimes x_{11112}^{14}+a_{4} x_{1}^{14} \otimes x_{1112}^{14} \\
& +a_{5} x_{1}^{28} \otimes x_{112}^{14}+a_{6} x_{1}^{42} \otimes x_{12}^{14} ; \\
& \underline{\Delta}\left(\left[x_{1112}, x_{112}\right]_{c}^{14}\right)=\left[x_{1112}, x_{112}\right]_{c}^{14} \otimes 1+1 \otimes\left[x_{1112}, x_{112}\right]_{c}^{14}+a_{7} x_{1112}^{14} \otimes x_{12}^{14} \\
& +a_{8} x_{11112}^{14} \otimes x_{12}^{14}+a_{9} x_{1}^{42} \otimes x_{12}^{28}+a_{10} x_{1}^{14} \otimes x_{112}^{28} \\
& +a_{11} x_{1}^{28} \otimes x_{12}^{14} x_{112}^{14}+a_{12} x_{1112}^{14} x_{1}^{14} \otimes x_{12}^{14} \text {; }
\end{aligned}
$$

with $a_{i} \in \mathbf{k}$. For instance,

$$
\begin{gathered}
a_{1}=q_{21}^{7}(1-\zeta)^{7}\left(1-\zeta^{5}\right)^{7}\left(4059-7124 \zeta+35105 \zeta^{2}+31472 \zeta^{3}-17431 \zeta^{4}\right. \\
\left.+19299 \zeta^{5}+40124 \zeta^{6}\right) \neq 0,
\end{gathered}
$$

because $\zeta \in \mathbb{G}_{7}^{\prime}$. Also, 


$$
\begin{gathered}
a_{3}=26686268+39070423 \zeta-42643895 \zeta^{2}-19103336 \zeta^{3}+52678504 \zeta^{4} \\
-4378676 \zeta^{5}-51111858 \zeta^{6} \neq 0 .
\end{gathered}
$$

Since $a_{1}, a_{3}, a_{6}, a_{12} \neq 0$ then $x_{112}^{14}, x_{1112}^{14}, x_{11112}^{14}$ and $\left[x_{1112}, x_{112}\right]_{c}^{14}$ are not primitive elements in $\widetilde{\mathcal{B}}_{\mathfrak{q}}$. Thus, $\xi_{1}$ and $\xi_{12}$ generates $\mathfrak{Z}_{\mathfrak{q}}$.

Also, in $\mathfrak{Z}_{\mathfrak{q}}$ we have

$$
\begin{array}{lr}
{\left[\xi_{12}, \xi_{1}\right]=a_{1} \xi_{112} ;} & {\left[\xi_{1}, \xi_{112}\right]=a_{2} \xi_{1112} ;} \\
{\left[\xi_{1}, \xi_{1112}\right]=a_{4} \xi_{11112} ;} & {\left[\xi_{1}, \xi_{11112}\right]=\left[\xi_{12}, \xi_{112}\right]=0 .}
\end{array}
$$

So, $\mathfrak{Z}_{\mathfrak{q}} \simeq \mathcal{U}\left(G_{2}^{+}\right)$.

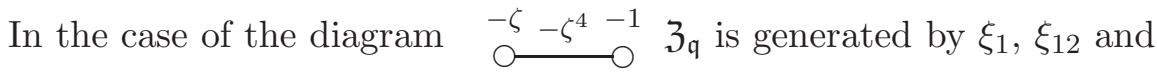

$$
\begin{aligned}
{\left[\xi_{12}, \xi_{1}\right] } & =b_{1} \xi_{112} ; & {\left[\xi_{12}, \xi_{112}\right] } & =b_{2} \xi_{112,12} ; \\
{\left[\xi_{12}, \xi_{112,12}\right] } & =b_{3} \xi_{(112,12), 12} ; & {\left[\xi_{1}, \xi_{112}\right] } & =\left[\xi_{12}, \xi_{(112,12), 12}\right]=0,
\end{aligned}
$$

where $b_{1}, b_{2}, b_{3} \in \mathbf{k}^{\times}$. Hence, we also have $\mathfrak{Z}_{\mathfrak{q}} \simeq \mathcal{U}\left(G_{2}^{+}\right)$.

Remark 4.1. The results of this paper are part of the thesis of one of the authors $[\mathrm{RB}$, where missing details of the computations can be found.

\section{REFERENCES}

[A] N. Andruskiewitsch, Notes on extensions of Hopf algebras. Canad. J. Math. 48 (1996), 3-42.

[AA] Andruskiewitsch, N; Angiono, I., Generalized root systems, contragredient Lie superalgebras and Nichols algebras, in preparation.

[AAR] Andruskiewitsch, N., Angiono, I., Rossi Bertone, F. The divided powers algebra of a finite-dimensional Nichols algebra of diagonal type, Math. Res. Lett., to appear.

[AAY] N. Andruskiewitsch, I. Angiono, H. Yamane. On pointed Hopf superalgebras, Contemp. Math. 544 (2011), 123-140.

[AN] N. Andruskiewitsch, S. Natale. Braided Hopf algebras arising from matched pairs of groups, J. Pure Appl. Alg. 182 (2003), 119-149.

[AS] N. Andruskiewitsch, H.-J. Schneider. Pointed Hopf algebras, New directions in Hopf algebras, MSRI series, Cambridge Univ. Press; 1-68 (2002).

[A1] I. Angiono. On Nichols algebras with standard braiding. Algebra and Number Theory, Vol. 3 (2009), 35-106.

[A2] I. Angiono. A presentation by generators and relations of Nichols algebras of diagonal type and convex orders on root systems. J. Eur. Math. Soc. 17 (2015), 2643-2671.

[A3] On Nichols algebras of diagonal type. J. Reine Angew. Math. 683 (2013), 189-251.

[A4] Nichols algebras of unidentified diagonal type, Comm. Alg 41 (2013), 46674693

[A5] Distinguished pre-Nichols algebras, Transform. Groups 21 (2016), 1-33.

[AY] I. Angiono, H. Yamane. The R-matrix of quantum doubles of Nichols algebras of diagonal type. J. Math. Phys. 56, 021702 (2015) 1-19.

[BD] Y. Bespalov, B. Drabant. Cross Product Bialgebras Part II, J. Algebra 240 (2001), $445-504$. 
[CH] M. Cuntz, I. Heckenberger. Weyl groupoids with at most three objects. J. Pure Appl. Algebra 213 (2009), 1112-1128.

[GG] J. Guccione, J. Guccione. Theory of braided Hopf crossed products, J. Algebra 261 (2003), 54-101.

[H1] I. Heckenberger. The Weyl groupoid of a Nichols algebra of diagonal type. Invent. Math. 164 (2006), 175-188.

[H2] Classification of arithmetic root systems. Adv. Math. 220 (2009), 59-124.

[H3] Lusztig isomorphisms for Drinfel'd doubles of bosonizations of Nichols algebras of diagonal type. J. Alg. 323 (2010), 2130-2180.

[K] V. Kharchenko, A quantum analogue of the Poincaré-Birkhoff-Witt theorem. Algebra and Logic 38 (1999), 259-276.

[L] G. Lusztig. Quantum groups at roots of 1 . Geom. Dedicata 35 (1990), 89-113.

[RB] F. Rossi Bertone. Álgebras cuánticas de potencias divididas. Tesis doctoral FaMAF, Universidad Nacional de Córdoba, available at www. famaf .unc. edu . ar/ rossib/.

[R] M. Rosso. Quantum groups and quantum shuffles. Inv. Math. 133 (1998), 399-416.

Famaf-Ciem (CONiCET), Universidad Nacional de Córdoba, Medina Allende s/n, Ciudad Universitaria, 5000 Córdoba, República Argentina.

E-mail address: (andrus|angiono|rossib) @mate.uncor.edu 